\title{
Co-evolutionary analysis suggests a role for TLR9 in papillomavirus restriction
}

3 Kelly King ${ }^{1}$, Brendan B. Larsen ${ }^{2}$, Sophie Gryseels ${ }^{2,3,4}$, Cécile Richet ${ }^{5}$, Simona Kraberger ${ }^{5}$, Robert

4 Jackson $^{1}$, Michael Worobey ${ }^{2,6}$, Joseph S. Harrison ${ }^{7}$, Arvind Varsani ${ }^{5,8}$, Koenraad Van

5 Doorslaer ${ }^{1,6,9 *}$

$7{ }^{1}$ School of Animal and Comparative Biomedical Sciences, University of Arizona, Tucson, AZ 8 85721, USA

$9{ }^{2}$ Department of Ecology and Evolutionary Biology, University of Arizona, Tucson, Arizona 85721, 10 USA.

$11{ }^{3}$ Department of Microbiology, Immunology and Transplantation, Rega Institute, KU Leuven, 3000

12 Leuven, Belgium.

$13{ }^{4}$ Department of Biology, University of Antwerp, 2000 Antwerp, Belgium.

$14{ }^{5}$ The Biodesign Center for Fundamental and Applied Microbiomics, Center for Evolution and

15 Medicine, School of Life Sciences, Arizona State University, Tempe, AZ 85287-5001, USA

$16{ }^{6}$ The BIO5 Institute, University of Arizona, Tucson, Arizona 85721, USA.

$17{ }^{7}$ Department of Chemistry, University of the Pacific, Stockton, California, USA.

$18{ }^{8}$ Structural Biology Research Unit, Department of Integrative Biomedical Sciences, University of

19 Cape Town, Observatory, Cape Town 7701, South Africa

$20{ }^{9}$ Department of Immunobiology; Cancer Biology Graduate Interdisciplinary Program; UA Cancer

21 Center, University of Arizona Tucson, AZ 85724, USA

23 * Correspondence: vandoorslaer@arizona.edu

25 Keywords: Papillomaviridae; Yinpterochiroptera; Yangochiroptera; Mexican free-tailed bat; 26 innate immunity, TLR9 


\section{A. Abstract}

28 Upon infection, DNA viruses can be sensed by pattern recognition receptors (PRRs) leading to

29 the activation of type I and III interferons, aimed at blocking infection. Therefore, viruses must

30 inhibit these signaling pathways, avoid being detected, or both. Papillomavirus virions are

31 trafficked from early endosomes to the Golgi apparatus and wait for the onset of mitosis to

32 complete nuclear entry. This unique subcellular trafficking strategy avoids detection by

33 cytoplasmic PRRs, a property that may contribute to establishment of infection. However, as the

34 capsid uncoats within acidic endosomal compartments, the viral DNA may be exposed to

35 detection by toll-like receptor (TLR) 9. In this study we characterize two new papillomaviruses

36 from bats and use molecular archeology to demonstrate that their genomes altered their

37 nucleotide composition to avoid detection by TLR9, providing evidence that TLR9 acts as a PRR

38 during papillomavirus infection. Furthermore, we demonstrate that TLR9, like other components

39 of the innate immune system, is under evolutionary selection in bats, providing the first direct

40 evidence for co-evolution between papillomaviruses and their hosts.

41 B. Introduction

42 Papillomaviruses (PVs) are circular double-stranded DNA viruses found in an extensive repertoire

43 of hosts, including mammals, reptiles, birds, and fish (Van Doorslaer 2013; Van Doorslaer et al.

$442013 ; 2017 ; 2018)$. In humans, roughly 400 genetically diverse papillomavirus types have been

45 described. While a subset of these viruses is associated with (malignant) tumors, most viral types

46 do not cause disease in immunocompetent hosts. As with humans, hosts that have been

47 thoroughly sampled are infected with an extensive repertoire of highly diverse yet species-specific

48 viruses. Co-evolution of virus and host alone is insufficient to explain the phylogeny of viruses in

49 the family Papillomaviridae. For example, papillomaviruses infecting humans do not form a

50 monophyletic group, within the papillomavirus family member phylogenetic tree suggests multiple

51 evolutionary mechanisms associated with host cellular interactions and immune evasion as 
52 important factors throughout viral genome evolution (Van Doorslaer 2013; Willemsen and Bravo 53 2019).

54 Furthermore, host or tissue tropism is likely a significant determinant of host-pathogen interactions 55 (Carey et al. 2019; Sawyer, Emerman, and Malik 2004; Taubenberger and Kash 2010). Therefore, 56 genomic analyses that consider essential mechanisms of the viral life cycle and evolutionary 57 pressures related to host-parasite interactions may provide a novel perspective into 58 papillomavirus genome evolution. A broader description of animal viruses will continue to inform 59 these efforts.

60 A successful infection requires that the papillomavirus DNA is delivered to the host cell nucleus.

61 Papillomaviruses access mitotically active basal cells through lesions in the stratified epithelia of 62 cutaneous or mucosal tissues. Following binding to cellular receptors and priming by kallikrein-8 63 and furin cleavage, the virus is endocytosed (Aksoy, Gottschalk, and Meneses 2017; Day and 64 Schelhaas 2014; DiGiuseppe, Bienkowska-Haba, Guion, and Sapp 2017; Richards et al. 2006;

65 Cerqueira et al. 2015; Day et al. 2013; Schelhaas et al. 2012). The viral DNA is transported to the 66 Golgi before the mitosis-dependent nuclear accumulation of L2 and viral DNA near PML bodies 67 (Day et al. 2013; Lipovsky et al. 2013; Popa et al. 2015; Aydin et al. 2017; 2014; Calton et al. 68 2017; DiGiuseppe, Bienkowska-Haba, Guion, Keiffer, et al. 2017; Stepp et al. 2017; Day et al. 69 2004).

70 Host cells detect a variety of viral pathogen-associated molecular patterns (PAMPs) by pattern71 recognition receptors (PRRs), resulting in induction of interferon (IFN) and a potent antiviral 72 response (Medzhitov 2007). The concerted actions of PRR signaling, specific viral-restriction 73 factors, and viral evasion strategies determine the eventual outcome of viral infection (Bowie and 74 Unterholzner 2008).

75 The papillomaviral structural proteins (L1 and L2) have no known enzymatic activity to directly 76 counteract antiviral responses (Buck, Day, and Trus 2013; J. W. Wang and Roden 2013). Instead, 77 we demonstrated that papillomaviruses evolved an elaborate trafficking mechanism to evade 
78 PRR sensing pathways within the cytosol (Uhlorn, Gamez, et al. 2020; Campos 2017; Uhlorn,

79 Jackson, et al. 2020). Furthermore, millions of years of virus-host co-speciation left historical

80 evidence of immune evasion events in these viruses' genomes (Sorouri et al. 2020). For example,

81 APOBEC3 has been demonstrated to restrict infection with HPV (Warren, Xu, et al. 2015; Warren,

82 Van Doorslaer, et al. 2015). We previously demonstrated that alphapapillomaviruses are

83 significantly depleted of TpC dinucleotides, the target for APOBEC3 mediated mutagenesis. This

$84 \mathrm{TpC}$ depletion evolved as a mechanism to evade APOBEC3 mediated mutagenesis. Specifically,

85 this depletion of the TpC content is more pronounced in mucosal alphapapillomaviruses and is

86 correlated with significantly higher expression levels of APOBEC3 in mucosal tissues (Warren,

87 Van Doorslaer, et al. 2015). These findings illustrate that host antiviral activity plays a critical role

88 in regulating papillomavirus evolution and that "molecular archeology" can be used to identify

89 these events.

90 Toll-like receptors (TLR) survey the extracellular and endosomal compartments and represent the

91 first defense line against foreign invaders. TLR2 and TLR4 recognize viral glycoproteins (Blanco

92 et al. 2010; Boehme, Guerrero, and Compton 2006; Jude et al. 2003; Bieback et al. 2002;

93 Murawski et al. 2009; Rassa et al. 2002; M. R. Thompson et al. 2011); TLR3 recognizes double-

94 stranded RNA (Alexopoulou et al. 2001; Bell et al. 2006; Gowen et al. 2006; Oshiumi et al. 2011;

95 F. Weber et al. 2006; Choe 2005), TLR7 and TLR8 recognize viral single-stranded RNA (Akira,

96 Uematsu, and Takeuchi 2006; Diebold 2004; Hemmi et al. 2002; Kawai and Akira 2006; Zucchini

97 et al. 2008; Jurk et al. 2002; Heil 2004). The endosomal TLR9 detects unmethylated CpG motifs

98 found in dsDNA (viral) genomes (Bowie and Unterholzner 2008; Gupta et al. 2015; M. R.

99 Thompson et al. 2011; J. Thompson and Iwasaki 2008).

100 As described above, HPV particles are endocytosed (Campos 2017; Calton et al. 2017), and viral

101 DNA could be recognized by endosomal TLR9 resulting in a downstream inflammatory immune

102 response. We hypothesize that TLR9 may detect papillomavirus dsDNA leading to CpG depletion,

103 similar to what we observed for APOBEC3 target motifs. Indeed, papillomavirus genomes have 
104 reduced CpG content (Warren, Van Doorslaer, et al. 2015; Upadhyay and Vivekanandan 2015).

105 This overall dinucleotide depletion confounds the ability to demonstrate the cause of this 106 depletion.

107 Bats serve as reservoirs for many viruses and and have served as the source of well-documented 108 cross-species transmission events of pathogens responsible for a myriad of epidemics, the most 109 notable being severe acute respiratory syndrome coronavirus 1 , middle east respiratory syndrome 110 coronavirus, Ebola virus, Marburg virus, and most recently, severe acute respiratory syndrome 111 coronavirus 2 (Banerjee et al. 2020; Brook and Dobson 2015; Wacharapluesadee et al. 2021). It

112 has been proposed that bats avoid immunopathological outcomes by not fully clearing a viral 113 infection (O'Shea et al. 2014). Indeed, bats have been reported to exhibit a 'dampened' immune 114 response to viral infections (Banerjee et al. 2020; Gorbunova, Seluanov, and Kennedy 2020; Xie 115 et al. 2018; Zhang et al. 2013; Subudhi, Rapin, and Misra 2019). The complex suppression of immune response pathways is variable between several bat species (order Chiroptera) (Zhang et

117 al. 2013; Jiang et al. 2017; Escalera-Zamudio et al. 2015; Hawkins et al. 2019). Importantly, it 118 was demonstrated that residues involved in the ligand-binding region of the bat TLR9 protein are 119 evolving under positive selection (Escalera-Zamudio et al. 2015; Jiang et al. 2017). This 120 evolutionary selection of TLR9 has been proposed to contribute to the high tolerance for viral 121 infections observed in bats (Banerjee et al. 2020; Hawkins et al. 2019). Notably, the theory of co122 evolution suggests that viruses need to counter these changes in TLR9, and we should be able 123 to detect these host-parasite interactions (Tan et al. 2017; Warren, Van Doorslaer, et al. 2015).

124 To address this question, we determined the genomes of two novel bat papillomaviruses from 125 Tadarida brasiliensis (TbraPV2, TbraPV3). Taxonomically, bats are classified into two suborders; 126 Yinpterochiroptera and Yangochiroptera. By comparing the genomes of papillomaviruses 127 associated with bats from either suborder, we demonstrate that TLR9 target motifs are 128 significantly depleted and impact papillomavirus evolution. Furthermore, we extend existing data 129 showing that Yangochiroptera TLR9 is evolving under diversifying selection. This argues that 
130 papillomavirus genomes are evolving in response to a host change. This is the first direct evidence

131 of PVs evolving in response to host evolutionary changes, thus providing direct evidence for co-

132 evolution. Also, these data argue that TLR9 is a restriction factor for papillomavirus infection.

134 C. Results

$135 \quad$ C1. Sampling, sample processing and viral metagenomics

136 We identified two circular contigs (circular based on terminal redundancy) that had similarities to 137 papillomavirus sequences. We mapped the raw reads to the assembled genomes using BBmap

138 (Bushnell 2014)to determine the read depth. For both the genomes we had a 22-25X coverage 139 depth across the whole genome with 1200-1300 mapped reads.

142 Using a metagenomics approach, we determined the genomes of two novel circular dsDNA

143 viruses. The open reading frames of these putative new viruses were determined using PuMA

144 (Pace et al., 2020). This analysis identified the typical papillomavirus open reading frames (E6,

$145 E 7, E 1, E 2, L 1$, and $L 2$ ) and the spliced $E 1^{\wedge} E 4$ and $E 8^{\wedge} E 2$ mRNAs), suggesting that we recovered

146 the genomes of two papillomaviruses associated with Mexican free-tailed bats (Tadarida

147 brasiliensis). The current papillomavirus taxonomy is based on sequence identity across the L1

148 open reading frame. If two viruses share more than $60 \%$, they fall into the same genus. Species

149 within a genus group viral 'types' that share between 70 and $90 \%$ sequence identity. A new 150 papillomavirus type shares less than $90 \%$ sequence identity with other viruses (Van Doorslaer et 151 al. 2018; Bernard et al. 2010; de Villiers et al. 2004). Both identified viruses share less than $90 \%$ 152 identity with their closest relatives (Figure 1B). In consultation with the international animal 153 papillomavirus reference center (Van Doorslaer and Dillner 2019), we name these two novel 154 papillomaviruses TbraPV2 (GenBank \# MW922427) and TbraPV3 (GenBank \# MW922428), 155 respectively. TbraPV2 is 8093 bp long, while TbraPV3 is 8037 bp long. Based on pairwise 
156 sequence identity in the L1 open reading frame, both viruses are most closely related to TbraPV1

157 (Figure 1B). TbraPV2 shares $81.7 \%$ sequence identity with TbraPV1 and likely represents a new 158 species in this as of yet unclassified genus. TbraPV3 shares $60.4 \%$ identity with TbraPV1, placing

159 it in the same genus. However, the phylogenetic tree shown in Figure 1 places TbraPV3 as an 160 outgroup to a clade that contains HPV41, EdPV1, TbraPV1, TbraPV2. Therefore, the evolutionary

161 history of these viruses does not support the current L1-based taxonomy.

162 It has been demonstrated that co-speciation between PVs and their hosts is a major contributor

163 to the papillomavirus's evolutionary history (Van Doorslaer 2013; Gottschling et al. 2007; 2011).

164 In support of this notion, these novel bat papillomaviruses cluster with other previously described

165 Chiropteran papillomaviruses (Figure 1A). However, as for other papillomavirus-host 166 relationships, bat-associated viruses are paraphyletic, suggesting that other evolutionary 167 mechanisms like intra-host divergence or niche adaptation likely contribute to the papillomavirus 168 phylogenetic tree (Buck et al. 2016; Van Doorslaer 2013).

169 C3. Chiropteran PVs co-speciated with their hosts

170 While TbraPV2 and TbraPV3 cluster together with several other Chiropteran viruses, the larger 171 clade consists of viruses infecting a wide array of mammals (red arrow in Figure 1A). In addition 172 to 6 species of Chiroptera, the subtree contains 16 host species classified in 5 mammalian orders. 173 We wanted to compare the evolutionary history of these diverse viruses to their hosts. Due to 174 intra-host divergence and niche adaptation, papillomaviruses infecting the same host can be 175 found in multiple phylogenetic tree clades. To ensure that viruses with a similar tissue tropism 176 and evolutionary history are compared, we extracted a subtree from the maximum likelihood tree 177 (Figure 2) (Smeele et al. 2018). This clade contains the newly identified TbraPV2 and TbraPV3 178 embedded within the largest monophyletic Chiroptera papillomavirus clade (red arrow in Figure 179 1A). We used a tanglegram to address our hypothesis of virus-host co-evolution (Figure 2A). In 180 this analysis, nodes in the host and virus phylogeny are rotated to optimize tip matching. 181 Similarities between the host and virus phylogenetic relationships are indicated by parallel lines 
182 linking the virus to its host in their respective trees. Conversely, mismatches in the evolutionary

183 history of the host and the virus show overlapping connecting lines. While there are some

184 overlapping connections between papillomaviruses and their hosts, most virus-host pairs support

185 the idea of co-speciation.

186 To formally quantify the degree of co-speciation, we focused on two datasets. First, we used the

187 phylogenetic tree shown in Figure 2. Because it was previously shown that members of the

188 Lambdapapillomavirus genus co-evolve with their hosts (Rector et al. 2007), we also tested a

189 smaller subtree to avoid skewing the results (indicated with the red arrow in Figure 2). The host

190 and virus phylogenetic trees were compared using the Wasserstein distance, estimated to be

1910.205 and 0.284 for the larger and smaller datasets, respectively. A Wasserstein distance of 0

192 indicates that both trees are topologically identical, while a value of 1 indicates complete lack of

193 congruence between both trees (Lewitus and Morlon 2016). Therefore, the host tree predicts the

194 virus tree, suggesting a role for co-speciation.

195 Also, we used the Procrustean Approach to Cophylogeny (PACo). This approach evaluates 196 congruency between distance matrices for each virus and associated host phylogenies

197 (Balbuena, Míguez-Lozano, and Blasco-Costa 2013; Hutchinson et al. 2017). The observed best-

198 fit Procrustean super-imposition (1.08E5 and 3.22E4 for the larger and smaller dataset,

199 respectively; denoted by the red dotted line) lies outside of the $95 \%$ confidence interval of the

200 ensemble of 1000 network randomizations in the null model (Figures 2B and C). Therefore, the

201 data allow us to reject the null hypotheses that the papillomavirus host tree does not predict the

202 virus tree and supports co-speciation as an important process for the evolution of this subclade

203 of PVs and their hosts (Balbuena, Míguez-Lozano, and Blasco-Costa 2013; Hutchinson et al.

204 2017).

205 C4. Yangochiropteran viruses have a reduced CpG content.

206 We previously demonstrated that millions of years of virus-host co-speciation left historical 207 evidence of this virus-host arms-race in the papillomavirus' genomes. For example, the mucosal, 
cancer-causing alphapapillomaviruses have a reduced TpC dinucleotide content, presumably due to evolutionary adaptations to APOBEC3 editing (Warren, Van Doorslaer, et al. 2015). To extend

210 these studies, we calculated the observed/predicted ratio for each dinucleotide in the viruses

211 shown in Figure 2. A ratio close to 1 indicates that a dinucleotide is seen in the sequence as often

212 as expected based on each sequence's nucleotide composition. Values lower than 1 suggest that

213 a dinucleotide is depleted. While the ApC, ApT, GpT, TpA, and TpC ratios are significantly lower

214 than 1 (one-sample t-test $p$-value $<0.001$ ), we observed the most significant decrease in the CpG

215 dinucleotide ratio (Figure 3). The median CpG content for these evolutionarily related viruses is

216 0.46. However, we noticed that the distribution has a long tail towards even lower values,

217 suggesting that some viruses have a further reduced $\mathrm{CpG}$ content (Figure 3).

218 When we plotted the CpG ratio for each virus on a phylogenetic tree (Figure 4A), it became clear

219 that the genomes of a subset of bat-associated papillomaviruses have a further decreased CpG

220 content. The order Chiroptera consists of two suborders, Yinpterochiroptera and Yangochiroptera

221 (Lei and Dong 2016; Teeling et al. 2002; Springer et al. 2001). When we associated the viruses

222 with their Yinpterochiroptera and Yangochiroptera hosts, the data demonstrates that the

223 Yangochiropteran papillomavirus genomes have even lower CpG values (orange bars in Figure

224 4A) when compared to the Yinpterochiroptera and other related papillomaviruses in the same 225 phylogenetic clade (blue bars) and members of a closely related clade (grey bars). Indeed, when 226 combined, the Yangochiropteran papillomavirus genomes have a significantly reduced CpG ratio 227 when compared to the other groups (Figure 4B ANOVA with posthoc Tukey test).

228 This reduction in the $\mathrm{CpG}$ ratio could be due to an overall lower GC content. We compared the 229 CpG ratio to total genomic GC content (Figure 4C). This analysis demonstrates that there is no 230 correlation between the decreased CpG ratio and the total GC content (linear regression: $R^{2}=$ $2310.008, p$-value $=0.27$, and the reduced $\mathrm{CpG}$ ratio is not simply due to a lower GC content. 
232 The viruses infecting bats in the Yangochiroptera suborder have a reduced CpG content, raising

233 the possibility that the host species is influencing the CpG ratio and evolutionary trajectory of

234 these viruses.

$235 \quad$ C5. CpG depletion alters codon usage without changing amino acid composition.

236 The $\mathrm{CpG}$ dinucleotide is present in 8 codons coding for five different amino acids. Therefore,

237 reducing $\mathrm{CpG}$ dinucleotides is expected to lead to a bias in codon usage or amino acid 238 composition. Roughly $85 \%$ of the papillomavirus genome codes for viral proteins (Van Doorslaer

239 et al. 2013; 2017). The viral genome contains several overlapping ORFs (Van Doorslaer 2013;

240 Van Doorslaer and McBride 2016). In some cases, this is a short overlap between the $3^{\prime}$ end of

241 one ORF and the 5' end of the downstream ORF (e.g., E6 and E7). In other cases, one ORF is

242 wholly embedded within the coding region of another ORF (e.g., E4 embedded in E2, or E8 within

243 E1). Since these overlapping regions are evolutionarily constrained at multiple codon positions

244 (i.e., codon position 3 in frame 1, would be codon position 2 in the overlapping frame (Miyata and

245 Yasunaga 1978), these overlapping regions were removed from the data (Materials and

246 Methods).

247 We determined codon usage tables for the non-overlapping coding sequences for each of the 248 papillomaviruses in the subtree described in Figure 2. These codon usage tables were compared 249 using the Emboss 'codcmp' tool to calculate codon usage differences. The more diverse the 250 codon usage, the larger the differences between both tables. This analysis shows that compared 251 to each other, the Yangochiroptera papillomaviruses codon usage is more similar than when other 252 viruses are compared (Figure 5A). This suggests that the reduction in CpG leads to a more 253 restricted availability of codons. The relative synonymous codon usage (RSCU) value is the ratio 254 of the observed frequency of one specific synonymous codon to the expected frequency (i.e., no 255 codon usage bias). This ratio is an important measure of codon usage bias (Sharp, Tuohy, and 256 Mosurski 1986). RSCU values higher than 1.6 and lower than 0.6 indicate overrepresented and 257 underrepresented codons, respectively (Wong et al. 2010). CpG containing codons (underlined) 
are significantly underrepresented in this dataset. For most amino-acids, CpG containing codons are further reduced in the Yangochiroptera papillomaviruses. Of note, in the case of arginine, the CpG containing codons are statistically significantly depleted when compared to the related

261 viruses (Figure 5C). Since these codons' CpGs are located in the $1^{\text {st }}$ and $2^{\text {nd }}$ position of the codon,

262 this depletion suggests that non-silent mutations are evolutionarily preferred over maintaining a 263 relatively high $\mathrm{CpG}$ content. Of note, despite the biased codon usage, there is no change in the 264 amino acid composition of the different viral proteins (Figure 5B). Thus, despite reducing CpG 265 content, the Yangochiroptera papillomaviruses are likely coding for similar proteins. These 266 observations likely explain the more restricted codon usage seen in Figure 5A.

267 C6. Natural selection in the TLR9 of Yangochiroptera bats.

268 Specific residues within the Chiropteran TLR9 have been demonstrated to be under positive 269 selective pressure (Escalera-Zamudio et al. 2015; Jiang et al. 2017). To determine whether 270 diversifying selection differentially affected Yangochiroptera and Yinpterochiroptera TLR9, we 271 constructed a maximum likelihood phylogenetic tree. As was previously reported, bat TLR9 272 sequences formed a monophyletic clade separate from other eutherian sequences (data not 273 shown), not as a sister-group to carnivores, ungulates, and cetaceans as is seen for other proteins 274 (Tsagkogeorga et al. 2013). We used RELAX (Wertheim et al. 2015), adaptive Branch Site 275 Relative Effect Likelihood (Smith et al. 2015), and Fixed effect Likelihood tests (Kosakovsky Pond 276 and Frost 2005) to detect evidence for evolutionary selection (materials and methods). RELAX 277 demonstrated that evolutionary selection intensified $(\mathrm{K}=6.07 ; \mathrm{LR}=21.05)$ within the 278 Yangochiroptera compared to the Yinpterochiroptera. Furthermore, aBSREL recovered evidence 279 for episodic diversifying selection on the branches leading to the Yangochiroptera (Figure 6B). 280 Finally, FEL identified 7 sites under diversifying selection within the Yangochiroptera.

281 We mapped a subset of these residues as well as residues previously identified to be under 282 diversifying selection (Escalera-Zamudio et al. 2015), and sites shown to be functionally important 283 for target recognition (Ohto et al. 2015) onto the structure of TLR9 bound to target DNA (Figure 
284 6A). Many of the sites are highly variable when compared to the mammalian consensus (Figure

285 6B). Notably, there are apparent differences between the TLR9 sequence of Yangochiroptera 286 and Yinpterochiroptera, specifically within the DNA recognition motif. In silico mutation analysis 287 suggests that these Yangochiroptera specific changes would alter how TLR9 recognizes its target

288 DNA. Overall, these mutations lead to a reduction in the positive surface charge of TLR9. Within 289 the Yangochiroptera, K51T leads to the loss of an ionic interaction with the phosphate backbone.

290 The Arg at position 76 is much larger than the canonical His at this position, which will presumably 291 impact the interaction with the DNA. While the P105 residue Vanderwaals bonds with the C6 and 292 T9 residues in the crystalized DNA, the 1105 is too bulky to occupy the same conformation as the 293 proline and will likely lead to a loss of the observed bend in the protein at this position. K181 is 294 involved in ionic interactions with the DNA backbone. Q181 has no charge and is too short to 295 interact with the DNA sidechain. E181 will likely charge repel the DNA backbone. Finally, K292 296 interacts with the DNA backbone, but this interaction in absent in Yangochiroptera TLR9 due to 297 the Ser residue at this position. Overall, the Yangochiroptera TLR9 DNA binding domain is 298 predicted to be functionally different from the Yinpterochiroptera and the other mammalian TLR9 299 molecules.

$300 \quad$ C7. CpG depletion points toward a TLR9 signature

301 Our data demonstrate that the Yangochiroptera TLR9 protein is under selective pressure, and 302 papillomaviruses that infect these hosts have a decreased CpG content. We hypothesized that 303 DNA recognition by TLR9 would lead to a decrease in CpG in the context of a TLR9 specific 304 PAMP. Thus, a specific (set of) tetramers should be depleted within the Yangochiroptera. We 305 calculated the observed/expected ratio for all tetramers and focused on those tetramers with a 306 central CpG (NCGN; Figure 7A). This initial analysis indicates that ACGT, GCGT, TCGA, and 307 TCGT are diminished in Yangochiroptera. We calculated the average tetramer ratio for each 308 group of viruses to normalize for the differences in overall CpG content between Yangochiroptera 309 and other viruses, as in Figure 4. We compared the proportion of Yang to Yin, Yang to other, and 
310 Yin to other (Figure 7B) for each tetramer. For example, in Yangochiroptera papillomaviruses,

311 'ACGT' is depleted three- to four-fold compared to other (brown) or Yinpterochiroptera

312 papillomaviruses (orange bar), respectively. Conversely, this tetramer is not depleted when

313 Yinpterochiroptera and other viruses are compared (blue bar). As expected, CpG containing

314 tetramers are depleted in Yangochiroptera papillomaviruses. Using a bootstrap method based on

3151000 randomly shuffled sequences (Materials and Methods), the 'ACGT' tetramer was identified

316 as significantly depleted within Yangochiroptera specific viruses (Figure 7C). This tetramer is

317 identical to the experimentally validated core mouse TLR9 recognition motif but is different from

318 the human TLR9 PAMP (TCGT) (Pohar et al. 2015; Krieg et al. 1995; Yi et al. 1998; G. Sen et al.

319 2004; Hartmann and Krieg 2000). This suggests that papillomaviruses associated with

320 Yangochiroptera specifically deplete CpG dinucleotides in the context of a known TLR9 PAMP.

321 D. Discussion

322 The data presented here advance our understanding of papillomavirus evolution and host-

323 pathogen interactions. Specifically, we provide evidence that papillomavirus genomes have

324 evolved to avoid detection by TLR9. The implications of this finding for papillomavirus biology are

325 discussed below.

326 D1. Viruses infecting bats in the suborder Yangochiroptera deplete CpG in a TLR9

327 dependent manner.

328 We demonstrate that the genomes of viruses isolated from specific bat species have a highly 329 reduced $\mathrm{CpG}$ content. A significant reduction of $\mathrm{CpG}$ sites in papillomavirus genomes has been 330 previously documented (Warren, Van Doorslaer, et al. 2015; Upadhyay and Vivekanandan 2015).

331 However, the reason for this depletion is unclear. Of note, in mammalian genomes, CpGs are

332 rare outside of so-called CpG islands (Illingworth and Bird 2009). This is believed to be mainly

333 due to the observation that methylated $\mathrm{CpGs}$ are prone to deamination, resulting in $\mathrm{C} \rightarrow \mathrm{T}$

334 mutations, leading to a depletion of CpG sites in the mammalian genomes over evolutionary time. 
335 Our data in Figure 3 show an increase in TpG and CpA. However, this increase does not appear

336 to be of the same magnitude as the dramatic reduction in $\mathrm{CpG}$ seen in the same dataset.

337 The zinc-finger antiviral protein (ZAP) acts as a broad-spectrum antiviral restriction protein that

338 recognizes $\mathrm{CpG}$ rich viral RNA, leading to RNA degradation and inhibition of translation (Gao

339 2002). Interestingly, it appears that ZAP exploits host CpG suppression to identify non-self RNA.

340 This may explain why multiple RNA viruses have reduced CpG content (Cheng et al. 2013;

341 Greenbaum et al. 2008), independently from CpG methylation as described above (Takata et al.

342 2017). ZAP was recently shown to restrict the replication of vaccinia virus Ankara (Peng et al.

343 2020) and HCMV (Lin et al. 2020), demonstrating that ZAP recognizes CpG rich viral RNA and

344 can restrict $\mathrm{CpG}$ rich DNA viruses. However, papillomavirus genomes are generally $\mathrm{CpG}$ depleted

345 (Warren, Van Doorslaer, et al. 2015; Upadhyay and Vivekanandan 2015). Furthermore, the

346 consensus recognition site for murine ZAP was identified as $\mathrm{CN}_{7}$ GNCG. In this motif, the CG

347 dinucleotide acts as the essential element, while the $\mathrm{G}$ further enhances binding affinity 10 -fold

348 (Luo et al. 2020). Our tetramer analysis does not identify a downregulation of GNCG in

349 Yangochiroptera specific papillomaviruses (data not shown). Therefore, it appears unlikely that

350 ZAP plays a vital role during papillomavirus infection. However, this would need to be

351 demonstrated experimentally.

352 In contrast, we provide evidence that the depletion of $\mathrm{CpG}$ in papillomavirus genomes is, at least

353 in part, due to the need to avoid detection by TLR9. Unmethylated CpG DNA motifs are

354 recognized by TLR9, leading to an interferon and inflammatory cytokine-mediated antiviral

355 response (Kawai and Akira 2006). By carefully analyzing the CpG content of related Chiropteran

356 papillomaviruses, we demonstrate that viruses isolated from Yangochiroptera have a further

357 decreased CpG content. Importantly, we demonstrate that the Yangochiroptera TLR9 protein is

358 evolving under diversifying selection, specifically sites implicated in DNA recognition. Finally, by

359 analyzing tetramer motifs, we show that Yangochiroptera are specifically depleted in ACGT, a

360 known TLR9 recognition motif. Together these data demonstrate that Yangochiroptera 
361 papillomaviruses deplete $\mathrm{CpG}$, in the context of a TLR9 recognition motif, presumably in response

362 to evolutionary changes within the TLR9 protein. This has important implications for 363 papillomavirus biology and evolution.

\section{D2. Recognition of papillomavirus DNA in the endosomes during infectious entry}

365 Shortly after entry, papillomavirus virions are trafficked from early endosomes into acidic late 366 endosome and multivesicular bodies, leading to capsid disassembly and uncoating viral DNA 367 (Campos 2017). Presumably, this exposes the viral DNA to TLR9, leading to an antiviral 368 response. Importantly, TLR9 specifically recognizes unmethylated CpG motifs. Several studies 369 have investigated the methylome of oncogenic human papillomaviruses (Johannsen and Lambert 370 2013). While these studies have demonstrated that the viral DNA is methylated under specific 371 conditions, it is unknown whether the packaged viral genome contains methylated CpG sites. 372 However, we have some clues that would suggest that viral DNA inside the virion is likely 373 hypomethylated. DNA methyltransferase 1 (DNMT1) is the primary cellular enzyme responsible 374 for maintaining DNA methylation patterns after replication. The DNMT1 protein was found 375 enriched in undifferentiated cells and is reduced as cells differentiate (G. L. Sen et al. 2010, 1).

376 Therefore, it is likely that the reduction in DNMT1 levels will lead to a loss of methylation on the 377 viral genomes destined for packaging and infection of the new tissue.

378 Differentially methylated CpG dinucleotides are present within consensus E2 binding sites in the 379 viral upstream regulatory region (McBride 2013). The binding of E2 to these binding sites is 380 important for viral replication, transcription, and proper partitioning of the viral genomes to 381 daughter cells (McBride 2013). In many viruses, the full-length E2 protein either activates or 382 represses viral transcription in a dose-dependent manner (Bouvard et al. 1994; Fujii et al. 2001; 383 Thierry and Yaniv 1987; Steger and Corbach 1997). CpG methylation of these sites inhibits E2 384 binding, presumably altering E2-mediated control of E6/E7 oncogene expression (Thain et al. 385 1997; Vinokurova and von Knebel Doeberitz 2011). However, the impact of changes to E2BS 386 methylation during cellular differentiation is not understood (Burley, Roberts, and Parish 2020). 
387 Nonetheless, studies using HPV16 containing cells suggest that the viral URR is hypomethylated 388 upon cellular differentiation (Kim et al. 2003).

389 A recent study showed that papillomavirus virions package DNA with histones enriched in 390 modifications typically associated with "active" (Porter et al. 2021). Of interest, the authors 391 demonstrate that the levels of $\mathrm{H} 3 \mathrm{~K} 4 \mathrm{me} 3$ were enriched on virions, compared to cellular controls 392 Conversely, virions were depleted in H3K9me3 (Porter et al. 2021). There is emerging evidence 393 of active associations between histone lysine methylation and DNA methylation (Rose and Klose 394 2014). For example, MeCP2 binds to methylated CpG (Nan et al. 1998), recruits the Suv39h1/2 395 histone methyltransferases (Fuks 2003), increasing H3K9me marks (Fuks 2003; Lunyak 2002). 396 In parallel, the $\mathrm{H} 3 \mathrm{~K} 9$ me mark recruits Dnmt3a/b to heterochromatin, leading to de novo 397 methylation of CpG sites (Lehnertz et al. 2003; Otani et al. 2009). Since H3K9me3 is depleted in 398 virions, it is tempting to conclude that virion DNA will be hypomethylated. Furthermore, H3K4me3 399 appears to be mutually exclusive with DNA methylation (M. Weber et al. 2007). H3K4me3 serves 400 as a binding site for H3K9me2 demethylases (Horton et al. 2010), which would lead to loss of 401 DNA methylation. Since virion DNA is enriched for $\mathrm{H} 3 \mathrm{~K} 4 \mathrm{me}$, this further strengthens the 402 hypothesis that viral DNA would be depleted in DNA methylation. Therefore, it seems reasonable 403 to assume that infecting the virus genome will be hypomethylated and therefore serve as a TLR9 404 PAMP.

405 As mentioned, recognition by TLR9 would lead to an antiviral response. Indeed, siRNA-mediated 406 knock-down of TLR9 has been shown to dramatically upregulate viral copy number and 407 transcription following infection with HPV16 (Hasan et al. 2013), suggesting that TLR9 can restrict 408 HPV infection. The observation that despite a reduction in viral CpG, HPV16 infection is still 409 improved by interfering with TLR9 (signaling) demonstrates an important rule in host-pathogen 410 interactions. While the loss of all (unmethylated) CpG dinucleotides would avoid detection by 411 TLR9, the virus can likely not completely remove all CpGs from its genome. The virus and the 412 host establish an uneasy balance. 


\section{D3. Sustained flight and the bat immune system}

414 Members of Chiroptera are classified into two suborders - Yinpterochiroptera (Rhinolophoid and

415 megabats) and Yangochiroptera all other bat species (Lei and Dong 2016; Teeling et al. 2002;

416 Springer et al. 2001). These suborders diverged roughly 60 million years ago (Lei and Dong

417 2016). Interestingly, the Yangochiroptera evolved flight and echolocation simultaneously, while

418 the Yinpterochiroptera evolved these features separately (Anderson and Ruxton 2020). Sustained

419 flight necessitated an increased metabolic capacity (Shen et al. 2010), which required bats to

420 accommodate oxidative metabolism by-products such as DNA damage (Barzilai 2002). Indeed,

421 many genes involved in DNA damage response and immunity have been demonstrated to be

422 under positive evolutionary selection (Hawkins et al. 2019; Zhang et al. 2013). Since both

423 suborders of bats 'invented' flight independently, likely, the corresponding adaptations are also

424 different. Indeed, we and others show that TLR9 is also under diversifying selection, specifically

425 in the Yangochiroptera. While TLR9 likely did not evolve specifically to restrict papillomavirus

426 infections, the virus likely needs to minimize its CpG content.

427 D4. Direct evidence of co-evolution between the virus and its hosts

428 Co-evolution alongside their hosts has been suggested to be an essential factor in the evolution

429 of papillomaviruses (Rector et al. 2007; Van Doorslaer 2013). However, the evolutionary history

430 of $\mathrm{PVs}$ is complex. PVs isolated from fish form a monophyletic group distinct from those from

431 mammals. However, within the mammalian papillomaviruses, there is no strict codivergence

432 pattern that would unambiguously indicate an ancient relationship between host and virus. There

433 has been no direct evidence in favor of co-evolution between papillomaviruses and their hosts.

434 The co-evolution theory would predict that the virus would need to adapt when the host evolves

435 a new skill. Therefore, as TLR9 evolves new functionalities, the virus would need to respond to

436 preserve the balance between virus and host. Indeed, our data suggest that as Yangochiroptera

437 TLR9 is undergoing diversifying selection, papillomavirus genomes infecting these bats further 
438 depleted their CpG content, specifically in the context of a known TLR9 PAMP. This is the first

439 direct evidence of co-evolution between this family of viruses and their hosts.

440 In the phylogenetic analysis, the viruses that infect Yinpterochiroptera and Yangochiroptera,

441 respectively, are not monophyletic but rather are present in three mixed clades (Figure 2; EsPV1,

442 EsPV3, and RfPV1; MscP2 and EhPV1; TbraPV1-3). This suggests that these three main clades

443 diverged before the ancestor of Yinpterochiroptera and Yangochiroptera split over 65 million years

444 ago. As these ancestral viruses co-evolved with the Yangochiroptera hosts, they selected for loss

445 of CpG. This occurred at least three separate times in the evolution of Yangochiroptera viruses.

446 This strongly argues against a founder effect but in favor of recurring co-evolutionary interactions.

\section{D5. Immune evasion by nucleotide sequence editing}

448 We previously used computer modeling and reconstruction of ancestral alphapapillomavirus 449 genomes to show that these viruses depleted TpC depletion to allow for replication in tissues with

450 high APOBEC3 expression - presumably to evade restriction by APOBEC3 by selecting for

451 variants that contain reduced target sites in their genomes. We observed a similar correlation

452 between TLR9 and CpG depletion, strengthening the notion that papillomaviruses avoid detection

453 by the immune system by changing the nucleotide composition of their genomes without

454 dramatically changing the protein-coding ability. This strategy likely allows the virus to maintain

455 its core functionalities. Most viral proteins are multifunctional and interact with a plethora of host

456 proteins. Amino acid level changes would likely disrupt these functions. The de novo evolution of

457 new proteins is rare and is further complicated by the small genome size and overlapping open

458 reading frames (Van Doorslaer and McBride 2016; Willemsen and Bravo 2019, 5).

459 D6. Oncogene mediated reduction of TLR9.

460 We propose that HPVs evade detection by TLR9 in the endosome by depleting CpG dinucleotides

461 from their genomes. Interestingly, the E6 and E7 oncoproteins of different human

462 papillomaviruses have been shown to downregulate the expression of TLR9 (Hasan et al. 2013;

463 2007; Pacini et al. 2015; 2017). Importantly, E6 and E7 are not delivered to the cell during infection 
464 but require onset of viral transcription after the viral genome is delivered to the nucleus and

465 presumably has already been sensed in the endosome. This implies that the ability to degrade

466 TLR9 may serve an additional function during the viral lifecycle, independent of initial infection.

467 This idea is supported by the observation that other viruses (Merkel Cell Polyomavirus, Hepatitis

$468 \mathrm{~B}$, and EBV) also interfere with TLR9 function during the maintenance phase of the infection

469 (Fathallah et al. 2010; Vincent et al. 2011; Shahzad et al. 2013). Nonetheless, this oncogene-

470 mediated repression of TLR9 occurs after infection and would still necessitate that the virus

471 evades detection during infectious entry.

\section{D7. Conclusion}

473 In conclusion, phylogenetic and genomic analyses of novel bat-associated viruses TbraPV2 and

474 TbraPV3 demonstrate that host-virus interaction, specifically evasion of the innate immune 475 system, affects the evolution of papillomaviruses. These data suggest that TLR9 acts as a 476 restriction factor for papillomavirus infection. Furthermore, we provide the first direct evidence for 477 co-evolution between papillomaviruses and their hosts.

478 E. Materials and Methods

479 E1. Data and Code availability

480 We retrieved full-length reference sequences from the PV database (PaVE; pave.niaid.nih.gov).

481 Data and code for all analyses is available from https://github.com/KVDlab/King-2021. TbraPV2 482 (MW922427) and TbraPV3 (MW922428) sequences are available on GenBank. Raw sequencing 483 data is available on SRA (PRJNA718335).

\section{E2. Sampling and sample processing}

485 Bats were captured in mist nets set over water sources, extracted from the nets and put in brown 486 paper bags. Bats were held in bags for 20 minutes, removed, and then measured and weighed. 487 Individuals were identified to species in the field using metrics such as forearm length and weight. 488 Feces and urine were collected from the bag or swabbed directly off the bat using a PurFlock 489 0.14" Ultrafine swab (Puritan, Guilford, Maine). All feces and urine samples were put into tubes 
containing $0.5 \mathrm{~mL}$ buffer consisting of $1 \times$ PBS and $50 \%$ Glycerol. These samples were held on

491 ice until returning to the lab where they were stored in a $-80^{\circ} \mathrm{C}$ freezer. All applicable international,

492 national and institutional guidelines for the care and use of animals were followed during

493 sampling. The study was approved by the University of Arizona Institutional Animal Care and Use

494 Committee permit \#15-583. Permits from the Arizona Department of Game and Fish were

495 numbered SP506475.

496 Of each of the fecal samples, $5 \mathrm{~g}$ was homogenized in SM buffer and the homogenate was

497 centrifuged at $6000 \times \mathrm{g}$ for $10 \mathrm{~min}$. The supernatant was sequentially filtered through $0.45 \mu \mathrm{m}$

498 and $0.2 \mu \mathrm{m}$ syringe filters and viral particles in the filtrate were precipitated with 15\% (w/v) PEG-

4998000 with overnight incubation at $4{ }^{\circ} \mathrm{C}$ followed by centrifugation at $10,000 \times \mathrm{g}$ as described

500 (Payne et al. 2020). The pellet was resuspended in $500 \mu \mathrm{L}$ of SM Buffer and $200 \mu \mathrm{L}$ of this was

501 used for viral DNA extraction using the High Pure Viral Nucleic Acid Kit (Roche Diagnostics,

502 Indianapolis, IN, USA). The total DNA was amplified using rolling circle amplification (RCA) with

503 the TempliPhi 2000 kit (GE Healthcare, USA) and the RCA products used to prepare Illumina

504 sequencing libraries then sequenced at Novagene Co. Ltd. (Hong Kong) on an Illumina NovaSeq

505 6000. The paired-end raw reads were trimmed using default settings within Trimmomatic v0.39

506 (Bolger, Lohse, and Usadel 2014) and the trimmed reads were de novo assembled using k-mer

507 values of 33,66 , and 77 within metaSPAdes $\vee 3.12 .0$ (Bankevich et al. 2012). The resulting

508 contigs greater than 500 nucleotides were analyzed by BLASTx (Altschul et al. 1990) against a

509 local viral protein database constructed from available NCBI RefSeq viral protein sequences

510 (https://ftp.ncbi.nlm.nih.gov/refseq/release/viral/).

\section{E3. Calculation of nucleotide frequencies}

512 To determine a single observed vs. expected (O/E) dinucleotide ratio across the entire viral 513 genome, a custom python script was used that leverages the CompSeq program from Emboss

514 (Warren, Van Doorslaer, et al. 2015). The expected frequencies of dinucleotide 'words' were 
515 estimated based on the observed frequency of single bases in the sequences. Only the forward

516 frame was analyzed.

517 The tetramer content for each genome was calculated as described for the dinucleotides. To

518 normalize tetramer content across groups of viruses we calculated the average O/E ratio across

519 the different groups. These average O/E ratios were compared as indicated in the figure legends.

520 To test whether any of the tested tetramers depletions are statistically significant, we randomly

521 shuffled each viral genome. To ensure that each randomly shuffled sequence would maintain the

522 same dinucleotide ratio as the original sequence, we used the Altschul and Erickson algorithm

523 (Stephen F Altschul and Erickson Bruce W 1985) as implemented by Clote and colleagues (Clote

524 2005). Based on these shuffled sequences, we calculated the above

525 Yangochiroptera/Yinpterochiroptera ratio. This was repeated 1000 times to establish a null

526 distribution. The 1-percentile was used as a significance cutoff.

\section{E4. Phylogenetic analyses}

528 Annotated sequences $(n=409)$ were downloaded from the PaVE genome database. A maximum

529 likelihood phylogenetic tree was constructed as described (King and Van Doorslaer 2018). The

530 amino-acid sequences for E1, E2, and L1 of all known papillomaviruses and the new TbraPV2

531 and TbraPV3 were individually aligned in MAFFT v7.3 (Katoh 2002; 2005) using the L-INS-I

532 algorithm. A partitioning scheme for the concatenated E1-E2-L1 alignment was determined under

533 corrected Akaike information criterion (AICc) implemented in PartitionFlnder2 (Lanfear et al.

534 2017), which separately identified each gene to evolve under the $L G+I+G+F$ evolutionary

535 substitution model. The concatenated E1-E2-L1 alignment was used to infer the best maximum

536 likelihood (ML) phylogenetic tree using RAxML-HPC v.8 (Stamatakis 2014) on CIPRES science

537 gateway (Miller, Pfeiffer, and Schwartz 2010) followed by a rapid bootstrapping analysis. A

538 posteriori bootstopping was automatically rendered in RAxML under the extended majority-rule

539 consensus tree criterion (autoMRE). The best ML tree was rendered and edited in RStudio using

540 the 'ggtree' (Yu et al. 2018) and 'treeio' (L.-G. Wang et al. 2020) packages. 
541 Taxonomic classification of TbraPV2 and TbraPV3 was based on pairwise sequence identity. The

542 L1 sequence of each pair was aligned at the amino acid level using the L-INS-I algorithm as

543 implemented within the MAFFT v7.3 (Katoh 2002; 2005). This way the alignments preserve the

544 codons. The resulting alignments are back translated to nucleotide alignments and used to

545 calculate pairwise sequence identity.

\section{E5. Coevolution analysis}

547 We used functions in the R 'ape' (Paradis and Schliep 2019) package to extract a well-supported 548 clade from the maximum likelihood phylogenetic tree. The extracted clade represents contains

549 viral sequences in the genera Lambdapapillomavirus, Mupapillomavirus, Nupapillomavirus, 550 Kappapapillomavirus, Sigmapapillomavirus, and Dyosigmapapillomavirus, and the largest set of

551 known of bat papillomaviruses, including the two novel bat papillomaviruses described in this 552 paper. A corresponding host species phylogeny was downloaded from TimeTree 553 (www.timetree.org) (Hedges, Dudley, and Kumar 2006; Kumar et al. 2017; Hedges et al. 2015).

554 A tanglegram representing the evolutionary relationship between the papillomaviruses and their 555 hosts was constructed in the 'phytools' package (Revell 2012). Phytools will optimize the 556 tanglegram by rotating nodes in the rooted phylogenies to minimize crossings between 557 connecting lines between both trees.

558 An additional subtree was extracted to minimize the impact of the genus Lamdapapillomavirus. 559 The viral types included in this smaller dataset are underlined in Figure 2. To assess the 560 congruency between PV and host phylogenies, we used the Procrustes Approach to 561 Cophylogenetic Analysis (PACo) (Balbuena, Míguez-Lozano, and Blasco-Costa 2013) as 562 implemented in R for both datasets. Briefly, PACo uses cophenetic distance matrices for the virus 563 and host trees and an association matrix of virus -host interactions. To assess statistical 564 significance, a Procrustean super-imposition of the sum of squared residuals was generated from 5651000 network randomizations under the "r2" randomization model. Under this model, host 566 specialization is assumed to drive the virus diversification (Hutchinson et al. 2017). The values 
567 for the actual tree comparisons were considered statistically significant if they fell outside the $95 \%$

568 confidence interval (C.I.)

569 To quantify the similarity between the virus and host phylogenies, we calculated the Wasserstein

570 distance using the 'castor' R package (Louca and Doebeli 2018). The Wasserstein distance is

571 based on a modified graph Laplacian (MGL). The MGL uses evolutionary distances between

572 nodes to construct a matrix which maintains branch length and tree topology information and

573 allows for the comparison of phylogenies from different species. Specifically, the differences

574 between a phylogeny's degree matrix (sum of branch lengths from one node $\mathrm{n}$ to all others) and

575 distance matrix (sum of all pairwise branch lengths) is calculated to generate a spectrum of

576 eigenvalues. To calculate a normalized MGL (nMGL) the MGL is divided by the degree matrix.

577 The normalized MGL is specifically useful when comparing trees on different timescales by

578 emphasizing topology over size (Lewitus and Morlon 2016). The Wasserstein distance represents

579 the largest eigenvalue from the spectra of the modified graph Laplacians. All eigenvalues from

580 the graph Laplacian spectrum were used to calculate the Wasserstein tree distance. The

581 Wasserstein tree distance metric calculated in 'castor' considers branch length and tree topology,

582 takes values between 0 and 1 . Identical tree topologies would have a Wasserstein distance of 0.

\section{E6. Analysis of codon usage}

584 A custom script was used to delete all overlaps between open reading frames. Briefly, overlaps

585 between E6 and E7, E1 and E8, E2 and E4, L2 and L1 were removed when present. For each

586 overlap, entire codons were removed as not introduce frameshifts. These sequences were

587 concatenated and further analyzed.

588 Cusp (Emboss suite of tools) was used to generate codon usage tables for each virus. These

589 tables were compared using codcmp (Emboss suite of tools). For each codon in the table codcmp

590 calculates the proportion of a codon to the total number of the codons in the. Next, codcmp

591 calculates the difference between the usage fractions in both tables.

592 The amino acid composition for each sequence was calculated as described (Carugo 2008). 


\section{E7. Diversifying selection of analysis of Yangochiroptera TLR9}

594 TLR9 sequences were downloaded from NCBI and translated into putative proteins. The amino

595 acid sequences were aligned using MAFFT v7.3, and back translated into codon-aware

596 nucleotide alignments. FastTree (Price, Dehal, and Arkin 2010) was used to construct a maximum

597 likelihood phylogenetic tree using the GTR substitution model of evolution.

598 To determine whether the strength of natural selection intensified along the along

599 Yangochiroptera compared to the Yinpterochiroptera, we used RELAX. After fitting a codon model

600 with three $\omega$ classes to the phylogeny (null model), RELAX then tests for changes to the intensity

601 of selection by introducing a selection parameter $k$. The null and alternative models are compared

602 using a Likelihood Ratio Test. A significant result of $k>1$ indicates that selection strength has been

603 intensified along the test branches (Wertheim et al. 2015).

604 aBSREL (adaptive Branch-Site Random Effects Likelihood) was used to test if positive selection

605 has occurred on the branches leading to Yangochiroptera. aBSREL determines whether a

606 proportion of sites have evolved under positive selection (Smith et al. 2015).

607 Finally, FEL (Fixed Effects Likelihood) was used to infer non-synonymous (dN) and synonymous

608 (dS) substitution rates on a per-site basis. This method assumes that the selection pressure for 609 each site is constant along the entire phylogeny. FEL fits a MG94xREV model to each codon site 610 to infer nonsynonymous and synonymous substitution rates at each site. A Likelihood Ratio Test 611 determines if $\mathrm{dN}$ is significantly greater than $\mathrm{dS}$.

\section{E8. Statistical analysis}

613 One- or two-way analysis of variance (ANOVA) were used where appropriate. Data are presented 614 as box-and-whisker plots with Tukey's method for outliers noted as distinct data points. All graphs 615 were generated using R. Results were considered statistically significant at a $P$-value of $<0.05$.

\section{References}


Akira, Shizuo, Satoshi Uematsu, and Osamu Takeuchi. 2006. "Pathogen Recognition and Innate Immunity." Cell 124 (4): 783-801. https://doi.org/10.1016/j.cell.2006.02.015.

Aksoy, Pinar, Elinor Y. Gottschalk, and Patricio I. Meneses. 2017. "HPV Entry into Cells." Mutation Research. Reviews in Mutation Research 772 (June): 13-22. https://doi.org/10.1016/j.mrrev.2016.09.004.

Alexopoulou, Lena, Agnieszka Czopik Holt, Ruslan Medzhitov, and Richard A. Flavell. 2001. "Recognition of Double-Stranded RNA and Activation of NF-KB by Toll-like Receptor 3." Nature 413 (6857): 732-38. https://doi.org/10.1038/35099560.

Altschul, S. F., W. Gish, W. Miller, E. W. Myers, and D. J. Lipman. 1990. "Basic Local Alignment Search Tool." Journal of Molecular Biology 215 (3): 403-10. https://doi.org/10.1016/S0022-2836(05)80360-2.

Anderson, Sophia C., and Graeme D. Ruxton. 2020. "The Evolution of Flight in Bats: A Novel Hypothesis." Mammal Review 50 (4): 426-39. https://doi.org/10.1111/mam.12211.

Aydin, Inci, Ruth Villalonga-Planells, Lilo Greune, Matthew P. Bronnimann, Christine M. Calton, Miriam Becker, Kun-Yi Lai, Samuel K. Campos, M. Alexander Schmidt, and Mario Schelhaas. 2017. "A Central Region in the Minor Capsid Protein of Papillomaviruses Facilitates Viral Genome Tethering and Membrane Penetration for Mitotic Nuclear Entry." PLoS Pathogens 13 (5): e1006308. https://doi.org/10.1371/journal.ppat.1006308.

Aydin, Inci, Susanne Weber, Berend Snijder, Pilar Samperio Ventayol, Andreas Kühbacher, Miriam Becker, Patricia M. Day, et al. 2014. "Large Scale RNAi Reveals the Requirement of Nuclear Envelope Breakdown for Nuclear Import of Human Papillomaviruses." PLoS Pathogens 10 (5): e1004162. https://doi.org/10.1371/journal.ppat.1004162.

Balbuena, Juan Antonio, Raúl Míguez-Lozano, and Isabel Blasco-Costa. 2013. "PACo: A Novel Procrustes Application to Cophylogenetic Analysis." PloS One 8 (4): e61048. https://doi.org/10.1371/journal.pone.0061048.

Banerjee, Arinjay, Michelle L. Baker, Kirsten Kulcsar, Vikram Misra, Raina Plowright, and Karen Mossman. 2020. "Novel Insights Into Immune Systems of Bats." Frontiers in Immunology 11: 26. https://doi.org/10.3389/fimmu.2020.00026.

Bankevich, Anton, Sergey Nurk, Dmitry Antipov, Alexey A. Gurevich, Mikhail Dvorkin, Alexander S. Kulikov, Valery M. Lesin, et al. 2012. "SPAdes: A New Genome Assembly Algorithm and Its Applications to Single-Cell Sequencing." Journal of Computational Biology 19 (5): 455-77. https://doi.org/10.1089/cmb.2012.0021.

Barzilai, A. 2002. "ATM Deficiency and Oxidative Stress: A New Dimension of Defective Response to DNA Damage." DNA Repair 1 (1): 3-25. https://doi.org/10.1016/S15687864(01)00007-6.

Bell, J. K., J. Askins, P. R. Hall, D. R. Davies, and D. M. Segal. 2006. “The DsRNA Binding Site of Human Toll-like Receptor 3." Proceedings of the National Academy of Sciences 103 (23): 8792-97. https://doi.org/10.1073/pnas.0603245103.

Bernard, Hans-Ulrich, Robert D. Burk, Zigui Chen, Koenraad van Doorslaer, Harald zur Hausen, and Ethel-Michele de Villiers. 2010. "Classification of Papillomaviruses (PVs) Based on 189 PV Types and Proposal of Taxonomic Amendments.” Virology 401 (1): 70-79. https://doi.org/10.1016/j.virol.2010.02.002.

Bieback, Karen, Egil Lien, Ingo M. Klagge, Elita Avota, Jürgen Schneider-Schaulies, W. Paul Duprex, Herrmann Wagner, Carsten J. Kirschning, Volker ter Meulen, and Sibylle 
Schneider-Schaulies. 2002. "Hemagglutinin Protein of Wild-Type Measles Virus Activates Toll-Like Receptor 2 Signaling.” Journal of Virology 76 (17): 8729-36. https://doi.org/10.1128/JVI.76.17.8729-8736.2002.

Blanco, Jorge C. G., Marina S. Boukhvalova, Kari A. Shirey, Gregory A. Prince, and Stefanie N. Vogel. 2010. "New Insights for Development of a Safe and Protective RSV Vaccine." Human Vaccines 6 (6): 482-92. https://doi.org/10.4161/hv.6.6.11562.

Boehme, Karl W., Mario Guerrero, and Teresa Compton. 2006. "Human Cytomegalovirus Envelope Glycoproteins B and H Are Necessary for TLR2 Activation in Permissive Cells." The Journal of Immunology 177 (10): 7094-7102. https://doi.org/10.4049/jimmunol.177.10.7094.

Bolger, Anthony M., Marc Lohse, and Bjoern Usadel. 2014. "Trimmomatic: A Flexible Trimmer for Illumina Sequence Data.” Bioinformatics 30 (15): 2114-20. https://doi.org/10.1093/bioinformatics/btu170.

Bouvard, V., A. Storey, D. Pim, and L. Banks. 1994. "Characterization of the Human Papillomavirus E2 Protein: Evidence of Trans-Activation and Trans-Repression in Cervical Keratinocytes." The EMBO Journal 13 (22): 5451-59.

Bowie, Andrew G., and Leonie Unterholzner. 2008. "Viral Evasion and Subversion of PatternRecognition Receptor Signalling." Nature Reviews. Immunology 8 (12): 911-22. https://doi.org/10.1038/nri2436.

Brook, Cara E., and Andrew P. Dobson. 2015. "Bats as 'Special' Reservoirs for Emerging Zoonotic Pathogens.” Trends in Microbiology 23 (3): 172-80. https://doi.org/10.1016/j.tim.2014.12.004.

Buck, Christopher B., Patricia M. Day, and Benes L. Trus. 2013. "The Papillomavirus Major Capsid Protein L1." Virology 445 (1-2): 169-74. https://doi.org/10.1016/j.virol.2013.05.038.

Buck, Christopher B., Koenraad Van Doorslaer, Alberto Peretti, Eileen M. Geoghegan, Michael J. Tisza, Ping An, Joshua P. Katz, et al. 2016. "The Ancient Evolutionary History of Polyomaviruses.” PLoS Pathogens 12 (4): e1005574. https://doi.org/10.1371/journal.ppat.1005574.

Burley, Megan, Sally Roberts, and Joanna L. Parish. 2020. "Epigenetic Regulation of Human Papillomavirus Transcription in the Productive Virus Life Cycle." Seminars in Immunopathology 42 (2): 159-71. https://doi.org/10.1007/s00281-019-00773-0.

Bushnell, Brian. 2014. "BBMap: A Fast, Accurate, Splice-Aware Aligner." https://www.osti.gov/biblio/1241166.

Calton, Christine M., Matthew P. Bronnimann, Ariana R. Manson, Shuaizhi Li, Janice A. Chapman, Marcela Suarez-Berumen, Tatum R. Williamson, Sudheer K. Molugu, Ricardo A. Bernal, and Samuel K. Campos. 2017. "Translocation of the Papillomavirus L2/VDNA Complex across the Limiting Membrane Requires the Onset of Mitosis." PLoS Pathogens 13 (5): e1006200. https://doi.org/10.1371/journal.ppat.1006200.

Campos, Samuel K. 2017. "Subcellular Trafficking of the Papillomavirus Genome during Initial Infection: The Remarkable Abilities of Minor Capsid Protein L2." Viruses 9 (12). https://doi.org/10.3390/v9120370.

Carey, Clayton M., Apurva A. Govande, Juliane M. Cooper, Melissa K. Hartley, Philip J. Kranzusch, and Nels C. Elde. 2019. "Recurrent Loss-of-Function Mutations Reveal Costs to OAS1 Antiviral Activity in Primates." Cell Host \& Microbe 25 (2): 336-343.e4. https://doi.org/10.1016/j.chom.2019.01.001. 
Carugo, Oliviero. 2008. "Amino Acid Composition and Protein Dimension.” Protein Science: A Publication of the Protein Society 17 (12): 2187-91. https://doi.org/10.1110/ps.037762.108. "Kallikrein-8 Proteolytically Processes Human Papillomaviruses in the Extracellular Space To Facilitate Entry into Host Cells.” Journal of Virology 89 (14): 7038-52. https://doi.org/10.1128/JVI.00234-15.

Cheng, Xiaofei, Nasar Virk, Wei Chen, Shuqin Ji, Shuxian Ji, Yuqiang Sun, and Xiaoyun Wu. 2013. "CpG Usage in RNA Viruses: Data and Hypotheses." Edited by Robert D. Burk. PLoS ONE 8 (9): e74109. https://doi.org/10.1371/journal.pone.0074109.

Choe, J. 2005. "Crystal Structure of Human Toll-Like Receptor 3 (TLR3) Ectodomain." Science 309 (5734): 581-85. https://doi.org/10.1126/science.1115253.

Clote, P. 2005. "Structural RNA Has Lower Folding Energy than Random RNA of the Same Dinucleotide Frequency.” RNA 11 (5): 578-91. https://doi.org/10.1261/rna.7220505.

Day, Patricia M., Carl C. Baker, Douglas R. Lowy, and John T. Schiller. 2004. "Establishment of Papillomavirus Infection Is Enhanced by Promyelocytic Leukemia Protein (PML) Expression." Proceedings of the National Academy of Sciences of the United States of America 101 (39): 14252-57. https://doi.org/10.1073/pnas.0404229101.

Day, Patricia M., and Mario Schelhaas. 2014. "Concepts of Papillomavirus Entry into Host Cells." Current Opinion in Virology 4 (February): 24-31. https://doi.org/10.1016/j.coviro.2013.11.002.

Day, Patricia M., Cynthia D. Thompson, Rachel M. Schowalter, Douglas R. Lowy, and John T. Schiller. 2013. "Identification of a Role for the Trans-Golgi Network in Human Papillomavirus 16 Pseudovirus Infection.” Journal of Virology 87 (7): 3862-70. https://doi.org/10.1128/JVI.03222-12.

Diebold, S. S. 2004. "Innate Antiviral Responses by Means of TLR7-Mediated Recognition of Single-Stranded RNA.” Science 303 (5663): 1529-31. https://doi.org/10.1126/science.1093616.

DiGiuseppe, Stephen, Malgorzata Bienkowska-Haba, Lucile G. M. Guion, Timothy R. Keiffer, and Martin Sapp. 2017. "Human Papillomavirus Major Capsid Protein L1 Remains Associated with the Incoming Viral Genome throughout the Entry Process." Journal of Virology 91 (16). https://doi.org/10.1128/JVI.00537-17.

DiGiuseppe, Stephen, Malgorzata Bienkowska-Haba, Lucile G. Guion, and Martin Sapp. 2017. "Cruising the Cellular Highways: How Human Papillomavirus Travels from the Surface to the Nucleus." Virus Research 231 (March): 1-9.

https://doi.org/10.1016/j.virusres.2016.10.015.

Escalera-Zamudio, Marina, M. Lisandra Zepeda-Mendoza, Elizabeth Loza-Rubio, Edith RojasAnaya, Maria L. Méndez-Ojeda, Carlos F. Arias, and Alex D. Greenwood. 2015. "The Evolution of Bat Nucleic Acid-Sensing Toll-like Receptors.” Molecular Ecology 24 (23): 5899-5909. https://doi.org/10.1111/mec.13431.

Fathallah, Ikbal, Peggy Parroche, Henri Gruffat, Claudia Zannetti, Hanna Johansson, Jiping Yue, Evelyn Manet, Massimo Tommasino, Bakary S. Sylla, and Uzma A. Hasan. 2010. "EBV Latent Membrane Protein 1 Is a Negative Regulator of TLR9." Journal of Immunology (Baltimore, Md.: 1950) 185 (11): 6439-47. https://doi.org/10.4049/jimmunol.0903459.

Fujii, Takuma, Janet L. Brandsma, Xueyan Peng, Srinivasan Srimatkandada, Lei Li, Allon Canaan, and Albert B. Deisseroth. 2001. "High and Low Levels of Cottontail Rabbit 
Papillomavirus E2 Protein Generate Opposite Effects on Gene Expression." Journal of Biological Chemistry 276 (2): 867-74. https://doi.org/10.1074/jbc.M007120200.

Fuks, F. 2003. "The DNA Methyltransferases Associate with HP1 and the SUV39H1 Histone Methyltransferase." Nucleic Acids Research 31 (9): 2305-12. https://doi.org/10.1093/nar/gkg332.

Gao, G. 2002. "Inhibition of Retroviral RNA Production by ZAP, a CCCH-Type Zinc Finger Protein.” Science 297 (5587): 1703-6. https://doi.org/10.1126/science.1074276.

Gorbunova, Vera, Andrei Seluanov, and Brian K. Kennedy. 2020. "The World Goes Bats: Living Longer and Tolerating Viruses." Cell Metabolism 32 (1): 31-43. https://doi.org/10.1016/j.cmet.2020.06.013.

Gottschling, Marc, Markus Göker, Alexandros Stamatakis, Olaf R. P. Bininda-Emonds, Ingo Nindl, and Ignacio G. Bravo. 2011. "Quantifying the Phylodynamic Forces Driving Papillomavirus Evolution.” Molecular Biology and Evolution 28 (7): 2101-13. https://doi.org/10.1093/molbev/msr030.

Gottschling, Marc, Alexandros Stamatakis, Ingo Nindl, Eggert Stockfleth, Angel Alonso, and Ignacio G. Bravo. 2007. "Multiple Evolutionary Mechanisms Drive Papillomavirus Diversification." Molecular Biology and Evolution 24 (5): 1242-58. https://doi.org/10.1093/molbev/msm039.

Gowen, Brian B., Justin D. Hoopes, Min-Hui Wong, Kie-Hoon Jung, Kevin C. Isakson, Lena Alexopoulou, Richard A. Flavell, and Robert W. Sidwell. 2006. "TLR3 Deletion Limits Mortality and Disease Severity Due to Phlebovirus Infection." The Journal of Immunology 177 (9): 6301-7. https://doi.org/10.4049/jimmunol.177.9.6301.

Greenbaum, Benjamin D., Arnold J. Levine, Gyan Bhanot, and Raul Rabadan. 2008. "Patterns of Evolution and Host Gene Mimicry in Influenza and Other RNA Viruses." Edited by Edward C. Holmes. PLoS Pathogens 4 (6): e1000079. https://doi.org/10.1371/journal.ppat.1000079.

Gupta, Chhedi Lal, Salman Akhtar, Andrew Waye, Nihar R. Pandey, Neelam Pathak, and Preeti Bajpai. 2015. "Cross Talk between Leishmania Donovani CpG DNA and Toll-like Receptor 9: An Immunoinformatics Approach." Biochemical and Biophysical Research Communications 459 (3): 424-29. https://doi.org/10.1016/j.bbrc.2015.02.121.

Hartmann, Gunther, and Arthur M. Krieg. 2000. "Mechanism and Function of a Newly Identified CpG DNA Motif in Human Primary B Cells.” The Journal of Immunology 164 (2): 94453. https://doi.org/10.4049/jimmunol.164.2.944.

Hasan, Uzma A., Elizabeth Bates, Fumihiko Takeshita, Alexandra Biliato, Rosita Accardi, Veronique Bouvard, Mariam Mansour, et al. 2007. "TLR9 Expression and Function Is Abolished by the Cervical Cancer-Associated Human Papillomavirus Type 16." The Journal of Immunology 178 (5): 3186-97. https://doi.org/10.4049/jimmunol.178.5.3186.

Hasan, Uzma A., Claudia Zannetti, Peggy Parroche, Nadège Goutagny, Marine Malfroy, Guillaume Roblot, Christine Carreira, et al. 2013. "The Human Papillomavirus Type 16 E7 Oncoprotein Induces a Transcriptional Repressor Complex on the Toll-like Receptor 9 Promoter." Journal of Experimental Medicine 210 (7): 1369-87. https://doi.org/10.1084/jem.20122394.

Hawkins, John A., Maria E. Kaczmarek, Marcel A. Müller, Christian Drosten, William H. Press, and Sara L. Sawyer. 2019. "A Metaanalysis of Bat Phylogenetics and Positive Selection Based on Genomes and Transcriptomes from 18 Species." Proceedings of the National Academy of Sciences 116 (23): 11351-60. https://doi.org/10.1073/pnas.1814995116. 
802

803

804

805

806

807

808

809

810

811

812

813

814

815

816

817

818

819

820

821

822

823

824

825

826

827

828

829

830

831

832

833

834

835

836

837

838

839

840

841

842

843

844

845

846

847
Hedges, S. Blair, Joel Dudley, and Sudhir Kumar. 2006. "TimeTree: A Public Knowledge-Base of Divergence Times among Organisms." Bioinformatics (Oxford, England) 22 (23): 2971-72. https://doi.org/10.1093/bioinformatics/bt1505.

Hedges, S. Blair, Julie Marin, Michael Suleski, Madeline Paymer, and Sudhir Kumar. 2015. "Tree of Life Reveals Clock-like Speciation and Diversification." Molecular Biology and Evolution 32 (4): 835-45. https://doi.org/10.1093/molbev/msv037.

Heil, F. 2004. "Species-Specific Recognition of Single-Stranded RNA via Toll-like Receptor 7 and 8." Science 303 (5663): 1526-29. https://doi.org/10.1126/science.1093620.

Hemmi, Hiroaki, Tsuneyasu Kaisho, Osamu Takeuchi, Shintaro Sato, Hideki Sanjo, Katsuaki Hoshino, Takao Horiuchi, Hideyuki Tomizawa, Kiyoshi Takeda, and Shizuo Akira. 2002. "Small Anti-Viral Compounds Activate Immune Cells via the TLR7 MyD88-Dependent Signaling Pathway." Nature Immunology 3 (2): 196-200. https://doi.org/10.1038/ni758.

Horton, John R, Anup K Upadhyay, Hank H Qi, Xing Zhang, Yang Shi, and Xiaodong Cheng. 2010. "Enzymatic and Structural Insights for Substrate Specificity of a Family of Jumonji Histone Lysine Demethylases." Nature Structural \& Molecular Biology 17 (1): 38-43. https://doi.org/10.1038/nsmb.1753.

Hutchinson, Matthew C., E. Fernando Cagua, Juan A. Balbuena, Daniel B. Stouffer, and Timothée Poisot. 2017. "Paco: Implementing Procrustean Approach to Cophylogeny in R.” Edited by Richard Fitzjohn. Methods in Ecology and Evolution 8 (8): 932-40. https://doi.org/10.1111/2041-210X.12736.

Illingworth, Robert S., and Adrian P. Bird. 2009. "CpG Islands - 'A Rough Guide.'” FEBS Letters 583 (11): 1713-20. https://doi.org/10.1016/j.febslet.2009.04.012.

Jiang, Haiying, Juan Li, Linmiao Li, Xiujuan Zhang, Lihong Yuan, and Jinping Chen. 2017. "Selective Evolution of Toll-like Receptors 3, 7, 8, and 9 in Bats." Immunogenetics 69 (4): 271-85. https://doi.org/10.1007/s00251-016-0966-2.

Johannsen, Eric, and Paul F. Lambert. 2013. "Epigenetics of Human Papillomaviruses." Virology 445 (1-2): 205-12. https://doi.org/10.1016/j.virol.2013.07.016.

Jude, Brooke A, Yelena Pobezinskaya, Jennifer Bishop, Susannah Parke, Ruslan M Medzhitov, Alexander V Chervonsky, and Tatyana V Golovkina. 2003. "Subversion of the Innate Immune System by a Retrovirus." Nature Immunology 4 (6): 573-78. https://doi.org/10.1038/ni926.

Jurk, Marion, Florian Heil, Jörg Vollmer, Christian Schetter, Arthur M. Krieg, Hermann Wagner, Grayson Lipford, and Stefan Bauer. 2002. "Human TLR7 or TLR8 Independently Confer Responsiveness to the Antiviral Compound R-848." Nature Immunology 3 (6): 499. https://doi.org/10.1038/ni0602-499.

Katoh, K. 2002. "MAFFT: A Novel Method for Rapid Multiple Sequence Alignment Based on Fast Fourier Transform." Nucleic Acids Research 30 (14): 3059-66. https://doi.org/10.1093/nar/gkf436.

- 2005. "MAFFT Version 5: Improvement in Accuracy of Multiple Sequence Alignment." Nucleic Acids Research 33 (2): 511-18. https://doi.org/10.1093/nar/gki198.

Kawai, Taro, and Shizuo Akira. 2006. "Innate Immune Recognition of Viral Infection." Nature Immunology 7 (2): 131-37. https://doi.org/10.1038/ni1303.

Kim, Kitai, Peggy A. Garner-Hamrick, Chris Fisher, Denis Lee, and Paul F. Lambert. 2003. "Methylation Patterns of Papillomavirus DNA, Its Influence on E2 Function, and Implications in Viral Infection." Journal of Virology 77 (23): 12450-59. https://doi.org/10.1128/jvi.77.23.12450-12459.2003. 
King, Kelly M., and Koenraad Van Doorslaer. 2018. "Building (Viral) Phylogenetic Trees Using a Maximum Likelihood Approach." Current Protocols in Microbiology 51 (1): e63. https://doi.org/10.1002/cpmc.63.

Kosakovsky Pond, Sergei L., and Simon D. W. Frost. 2005. "Not So Different After All: A Comparison of Methods for Detecting Amino Acid Sites Under Selection." Molecular Biology and Evolution 22 (5): 1208-22. https://doi.org/10.1093/molbev/msil05.

Krieg, Arthur M., Ae-Kyung Yi, Sara Matson, Thomas J. Waldschmidt, Gail A. Bishop, Rebecca Teasdale, Gary A. Koretzky, and Dennis M. Klinman. 1995. "CpG Motifs in Bacterial DNA Trigger Direct B-Cell Activation.” Nature 374 (6522): 546-49. https://doi.org/10.1038/374546a0.

Kumar, Sudhir, Glen Stecher, Michael Suleski, and S. Blair Hedges. 2017. "TimeTree: A Resource for Timelines, Timetrees, and Divergence Times.” Molecular Biology and Evolution 34 (7): 1812-19. https://doi.org/10.1093/molbev/msx116.

Lanfear, Robert, Paul B. Frandsen, April M. Wright, Tereza Senfeld, and Brett Calcott. 2017. "PartitionFinder 2: New Methods for Selecting Partitioned Models of Evolution for Molecular and Morphological Phylogenetic Analyses." Molecular Biology and Evolution 34 (3): 772-73. https://doi.org/10.1093/molbev/msw260.

Lehnertz, Bernhard, Yoshihide Ueda, Alwin A.H.A. Derijck, Ulrich Braunschweig, Laura PerezBurgos, Stefan Kubicek, Taiping Chen, En Li, Thomas Jenuwein, and Antoine H.F.M. Peters. 2003. "Suv39h-Mediated Histone H3 Lysine 9 Methylation Directs DNA Methylation to Major Satellite Repeats at Pericentric Heterochromatin." Current Biology 13 (14): 1192-1200. https://doi.org/10.1016/S0960-9822(03)00432-9.

Lei, Ming, and Dong Dong. 2016. "Phylogenomic Analyses of Bat Subordinal Relationships Based on Transcriptome Data." Scientific Reports 6 (1): 27726. https://doi.org/10.1038/srep27726.

Lewitus, Eric, and Helene Morlon. 2016. "Characterizing and Comparing Phylogenies from Their Laplacian Spectrum.” Systematic Biology 65 (3): 495-507. https://doi.org/10.1093/sysbio/syv116.

Lin, Yao-Tang, Stephen Chiweshe, Dominique McCormick, Anna Raper, Arthur Wickenhagen, Victor DeFillipis, Eleanor Gaunt, Peter Simmonds, Sam J. Wilson, and Finn Grey. 2020. "Human Cytomegalovirus Evades ZAP Detection by Suppressing CpG Dinucleotides in the Major Immediate Early 1 Gene.” Edited by Eain A. Murphy. PLOS Pathogens 16 (9): e1008844. https://doi.org/10.1371/journal.ppat.1008844.

Lipovsky, Alex, Andreea Popa, Genaro Pimienta, Michael Wyler, Ashima Bhan, Leena Kuruvilla, Marie-Aude Guie, et al. 2013. "Genome-Wide SiRNA Screen Identifies the Retromer as a Cellular Entry Factor for Human Papillomavirus." Proceedings of the National Academy of Sciences of the United States of America 110 (18): 7452-57. https://doi.org/10.1073/pnas.1302164110.

Louca, Stilianos, and Michael Doebeli. 2018. "Efficient Comparative Phylogenetics on Large Trees." Bioinformatics (Oxford, England) 34 (6): 1053-55. https://doi.org/10.1093/bioinformatics/btx701.

Lunyak, V. V. 2002. "Corepressor-Dependent Silencing of Chromosomal Regions Encoding Neuronal Genes.” Science 298 (5599): 1747-52. https://doi.org/10.1126/science.1076469. 
934

935

936

Luo, Xiu, Xinlu Wang, Yina Gao, Jingpeng Zhu, Songqing Liu, Guangxia Gao, and Pu Gao. 2020. "Molecular Mechanism of RNA Recognition by Zinc-Finger Antiviral Protein." Cell Reports 30 (1): 46-52.e4. https://doi.org/10.1016/j.celrep.2019.11.116.

McBride, Alison A. 2013. "The Papillomavirus E2 Proteins." Virology 445 (1-2): 57-79. https://doi.org/10.1016/j.virol.2013.06.006.

Medzhitov, Ruslan. 2007. "Recognition of Microorganisms and Activation of the Immune Response.” Nature 449 (7164): 819-26. https://doi.org/10.1038/nature06246.

Miller, Mark A., Wayne Pfeiffer, and Terri Schwartz. 2010. "Creating the CIPRES Science Gateway for Inference of Large Phylogenetic Trees." In 2010 Gateway Computing Environments Workshop (GCE), 1-8. New Orleans, LA, USA: IEEE. https://doi.org/10.1109/GCE.2010.5676129.

Miyata, T., and T. Yasunaga. 1978. "Evolution of Overlapping Genes.” Nature 272 (5653): 53235. https://doi.org/10.1038/272532a0.

Murawski, Matthew R., Glennice N. Bowen, Anna M. Cerny, Larry J. Anderson, Lia M. Haynes, Ralph A. Tripp, Evelyn A. Kurt-Jones, and Robert W. Finberg. 2009. "Respiratory Syncytial Virus Activates Innate Immunity through Toll-Like Receptor 2." Journal of Virology 83 (3): 1492-1500. https://doi.org/10.1128/JVI.00671-08.

Nan, X., H. H. Ng, C. A. Johnson, C. D. Laherty, B. M. Turner, R. N. Eisenman, and A. Bird. 1998. "Transcriptional Repression by the Methyl-CpG-Binding Protein MeCP2 Involves a Histone Deacetylase Complex.” Nature 393 (6683): 386-89. https://doi.org/10.1038/30764.

Ohto, Umeharu, Takuma Shibata, Hiromi Tanji, Hanako Ishida, Elena Krayukhina, Susumu Uchiyama, Kensuke Miyake, and Toshiyuki Shimizu. 2015. "Structural Basis of CpG and Inhibitory DNA Recognition by Toll-like Receptor 9.” Nature 520 (7549): 702-5. https://doi.org/10.1038/nature14138.

O'Shea, Thomas J., Paul M. Cryan, Andrew A. Cunningham, Anthony R. Fooks, David T.S. Hayman, Angela D. Luis, Alison J. Peel, Raina K. Plowright, and James L.N. Wood. 2014. "Bat Flight and Zoonotic Viruses." Emerging Infectious Diseases 20 (5): 741-45. https://doi.org/10.3201/eid2005.130539.

Oshiumi, Hiroyuki, Masaaki Okamoto, Ken Fujii, Takashi Kawanishi, Misako Matsumoto, Satoshi Koike, and Tsukasa Seya. 2011. "The TLR3/TICAM-1 Pathway Is Mandatory for Innate Immune Responses to Poliovirus Infection.” The Journal of Immunology 187 (10): 5320-27. https://doi.org/10.4049/jimmunol.1101503.

Otani, Junji, Toshiyuki Nankumo, Kyohei Arita, Susumu Inamoto, Mariko Ariyoshi, and Masahiro Shirakawa. 2009. "Structural Basis for Recognition of H3K4 Methylation Status by the DNA Methyltransferase 3A ATRX-DNMT3-DNMT3L Domain." EMBO Reports 10 (11): 1235-41. https://doi.org/10.1038/embor.2009.218.

Pacini, Laura, Maria Grazia Ceraolo, Assunta Venuti, Giusi Melita, Uzma A. Hasan, Rosita Accardi, and Massimo Tommasino. 2017. "UV Radiation Activates Toll-Like Receptor 9 Expression in Primary Human Keratinocytes, an Event Inhibited by Human Papillomavirus 38 E6 and E7 Oncoproteins.” Journal of Virology 91 (19). https://doi.org/10.1128/JVI.01123-17.

Pacini, Laura, Claudia Savini, Raffaella Ghittoni, Djamel Saidj, Jerome Lamartine, Uzma A. Hasan, Rosita Accardi, and Massimo Tommasino. 2015. "Downregulation of Toll-Like Receptor 9 Expression by Beta Human Papillomavirus 38 and Implications for Cell Cycle 
Control.” Edited by R. M. Sandri-Goldin. Journal of Virology 89 (22): 11396-405. https://doi.org/10.1128/JVI.02151-15.

Paradis, Emmanuel, and Klaus Schliep. 2019. "Ape 5.0: An Environment for Modern Phylogenetics and Evolutionary Analyses in R." Bioinformatics (Oxford, England) 35 (3): 526-28. https://doi.org/10.1093/bioinformatics/bty633.

Payne, Natalie, Simona Kraberger, Rafaela S. Fontenele, Kara Schmidlin, Melissa H. Bergeman, Ivonne Cassaigne, Melanie Culver, Arvind Varsani, and Koenraad Van Doorslaer. 2020. "Novel Circoviruses Detected in Feces of Sonoran Felids." Viruses 12 (9). https://doi.org/10.3390/v12091027.

Peng, Chen, Linda S. Wyatt, Shira G. Glushakow-Smith, Madhu Lal-Nag, Andrea S. Weisberg, and Bernard Moss. 2020. "Zinc-Finger Antiviral Protein (ZAP) Is a Restriction Factor for Replication of Modified Vaccinia Virus Ankara (MVA) in Human Cells." Edited by Matthew S. Wiebe. PLOS Pathogens 16 (8): e1008845. https://doi.org/10.1371/journal.ppat.1008845.

Pohar, Jelka, Duško Lainšček, Ryutaro Fukui, Chikako Yamamoto, Kensuke Miyake, Roman Jerala, and Mojca Benčina. 2015. "Species-Specific Minimal Sequence Motif for Oligodeoxyribonucleotides Activating Mouse TLR9.” The Journal of Immunology 195 (9): 4396-4405. https://doi.org/10.4049/jimmunol.1500600.

Popa, Andreea, Wei Zhang, Megan S. Harrison, Kylia Goodner, Teymur Kazakov, Edward C. Goodwin, Alex Lipovsky, Christopher G. Burd, and Daniel DiMaio. 2015. "Direct Binding of Retromer to Human Papillomavirus Type 16 Minor Capsid Protein L2 Mediates Endosome Exit during Viral Infection.” PLoS Pathogens 11 (2): e1004699. https://doi.org/10.1371/journal.ppat.1004699.

Porter, Samuel S., Jennifer C. Liddle, Kristen Browne, Diana V. Pastrana, Benjamin A. Garcia, Christopher B. Buck, Matthew D. Weitzman, and Alison A. McBride. 2021. "Histone Modifications in Papillomavirus Virion Minichromosomes." Edited by Thomas Shenk. MBio 12 (1): e03274-20,/mbio/12/1/mBio.03274-20.atom. https://doi.org/10.1128/mBio.03274-20.

Price, Morgan N., Paramvir S. Dehal, and Adam P. Arkin. 2010. "FastTree 2 - Approximately Maximum-Likelihood Trees for Large Alignments.” Edited by Art F. Y. Poon. PLoS ONE 5 (3): e9490. https://doi.org/10.1371/journal.pone.0009490.

Rassa, John C., Jennifer L. Meyers, Yuanming Zhang, Rama Kudaravalli, and Susan R. Ross. 2002. "Murine Retroviruses Activate B Cells via Interaction with Toll-like Receptor 4." Proceedings of the National Academy of Sciences of the United States of America 99 (4): 2281-86. https://doi.org/10.1073/pnas.042355399.

Rector, Annabel, Philippe Lemey, Ruth Tachezy, Sara Mostmans, Shin-Je Ghim, Koenraad Van Doorslaer, Melody Roelke, et al. 2007. "Ancient Papillomavirus-Host Co-Speciation in Felidae.” Genome Biology 8 (4): R57. https://doi.org/10.1186/gb-2007-8-4-r57.

Revell, Liam J. 2012. "Phytools: An R Package for Phylogenetic Comparative Biology (and Other Things): Phytools: R Package." Methods in Ecology and Evolution 3 (2): 217-23. https://doi.org/10.1111/j.2041-210X.2011.00169.x.

Richards, Rebecca M., Douglas R. Lowy, John T. Schiller, and Patricia M. Day. 2006. "Cleavage of the Papillomavirus Minor Capsid Protein, L2, at a Furin Consensus Site Is Necessary for Infection." Proceedings of the National Academy of Sciences of the United States of America 103 (5): 1522-27. https://doi.org/10.1073/pnas.0508815103. 
982

983

984

985

986

987

988

989

990

991

992

993

994

995

996

997

998

999

1000

1001

1002

1003

1004

1005

1006

1007

1008

1009

1010

1011

1012

1013

1014

1015

1016

1017

1018

1019

1020

1021

1022

1023

1024

1025

1026

1027
Rose, Nathan R., and Robert J. Klose. 2014. "Understanding the Relationship between DNA Methylation and Histone Lysine Methylation." Biochimica et Biophysica Acta (BBA) Gene Regulatory Mechanisms 1839 (12): 1362-72. https://doi.org/10.1016/j.bbagrm.2014.02.007.

Sawyer, Sara L., Michael Emerman, and Harmit S. Malik. 2004. "Ancient Adaptive Evolution of the Primate Antiviral DNA-Editing Enzyme APOBEC3G.” PLoS Biology 2 (9): E275. https://doi.org/10.1371/journal.pbio.0020275.

Schelhaas, Mario, Bhavin Shah, Michael Holzer, Peter Blattmann, Lena Kühling, Patricia M. Day, John T. Schiller, and Ari Helenius. 2012. "Entry of Human Papillomavirus Type 16 by Actin-Dependent, Clathrin- and Lipid Raft-Independent Endocytosis." PLoS Pathogens 8 (4): e1002657. https://doi.org/10.1371/journal.ppat.1002657.

Sen, George L., Jason A. Reuter, Daniel E. Webster, Lilly Zhu, and Paul A. Khavari. 2010. "DNMT1 Maintains Progenitor Function in Self-Renewing Somatic Tissue." Nature 463 (7280): 563-67. https://doi.org/10.1038/nature08683.

Sen, Goutam, Michael Flora, Gouri Chattopadhyay, Dennis M. Klinman, Andrew Lees, James J. Mond, and Clifford M. Snapper. 2004. "The Critical DNA Flanking Sequences of a CpG Oligodeoxynucleotide, but Not the 6 Base CpG Motif, Can Be Replaced with RNA without Quantitative or Qualitative Changes in Toll-like Receptor 9-Mediated Activity." Cellular Immunology 232 (1-2): 64-74. https://doi.org/10.1016/j.cellimm.2005.01.010.

Shahzad, Naveed, Masahiro Shuda, Tarik Gheit, Hyun Jin Kwun, Iris Cornet, Djamel Saidj, Claudia Zannetti, et al. 2013. "The T Antigen Locus of Merkel Cell Polyomavirus Downregulates Human Toll-like Receptor 9 Expression.” Journal of Virology 87 (23): 13009-19. https://doi.org/10.1128/JVI.01786-13.

Sharp, Paul M., Therese M.F. Tuohy, and Krzysztof R. Mosurski. 1986. "Codon Usage in Yeast: Cluster Analysis Clearly Differentiates Highly and Lowly Expressed Genes." Nucleic Acids Research 14 (13): 5125-43. https://doi.org/10.1093/nar/14.13.5125.

Shen, Y.-Y., L. Liang, Z.-H. Zhu, W.-P. Zhou, D. M. Irwin, and Y.-P. Zhang. 2010. “Adaptive Evolution of Energy Metabolism Genes and the Origin of Flight in Bats." Proceedings of the National Academy of Sciences 107 (19): 8666-71. https://doi.org/10.1073/pnas.0912613107.

Smeele, Zoe E., Jennifer M. Burns, Koenraad Van Doorsaler, Rafaela S. Fontenele, Kara Waits, Daisy Stainton, Michelle R. Shero, et al. 2018. "Diverse Papillomaviruses Identified in Weddell Seals." The Journal of General Virology, February. https://doi.org/10.1099/jgv.0.001028.

Smith, Martin D., Joel O. Wertheim, Steven Weaver, Ben Murrell, Konrad Scheffler, and Sergei L. Kosakovsky Pond. 2015. "Less Is More: An Adaptive Branch-Site Random Effects Model for Efficient Detection of Episodic Diversifying Selection." Molecular Biology and Evolution 32 (5): 1342-53. https://doi.org/10.1093/molbev/msv022.

Sorouri, Mahsa, Tyron Chang, Palmy Jesudhasan, Chelsea Pinkham, Nels C. Elde, and Dustin C. Hancks. 2020. "Signatures of Host-Pathogen Evolutionary Conflict Reveal MISTR-A Conserved MItochondrial STress Response Network.” Edited by Mark L Siegal. PLOS Biology 18 (12): e3001045. https://doi.org/10.1371/journal.pbio.3001045.

Springer, M. S., E. C. Teeling, O. Madsen, M. J. Stanhope, and W. W. de Jong. 2001. "Integrated Fossil and Molecular Data Reconstruct Bat Echolocation." Proceedings of the National Academy of Sciences 98 (11): 6241-46. https://doi.org/10.1073/pnas.111551998. 
1028

1029

1030

1031

1032

1033

1034

1035

1036

1037

1038

1039

1040

1041

1042

1043

1044

1045

1046

1047

1048

1049

1050

1051

1052

1053

1054

1055

1056

1057

1058

1059

1060

1061

1062

1063

1064

1065

1066

1067

1068

1069

1070

1071

1072
Stamatakis, Alexandros. 2014. "RAxML Version 8: A Tool for Phylogenetic Analysis and PostAnalysis of Large Phylogenies.” Bioinformatics 30 (9): 1312-13. https://doi.org/10.1093/bioinformatics/btu033.

Steger, G, and S Corbach. 1997. "Dose-Dependent Regulation of the Early Promoter of Human Papillomavirus Type 18 by the Viral E2 Protein.” Journal of Virology 71 (1): 50-58. https://doi.org/10.1128/JVI.71.1.50-58.1997.

Stephen F Altschul and Erickson Bruce W. 1985. "Significance of Nucleotide Sequence Alignments: A Method for Random Sequence Permutation That Preserves Dinucleotide and Codon Usage." Molecular Biology and Evolution, November. https://doi.org/10.1093/oxfordjournals.molbev.a040370.

Stepp, Wesley H., James D. Stamos, Simran Khurana, Alix Warburton, and Alison A. McBride. 2017. "Sp100 Colocalizes with HPV Replication Foci and Restricts the Productive Stage of the Infectious Cycle." Edited by Paul Francis Lambert. PLOS Pathogens 13 (10): e1006660. https://doi.org/10.1371/journal.ppat.1006660.

Subudhi, Sonu, Noreen Rapin, and Vikram Misra. 2019. "Immune System Modulation and Viral Persistence in Bats: Understanding Viral Spillover.” Viruses 11 (2). https://doi.org/10.3390/v11020192.

Takata, Matthew A., Daniel Gonçalves-Carneiro, Trinity M. Zang, Steven J. Soll, Ashley York, Daniel Blanco-Melo, and Paul D. Bieniasz. 2017. "CG Dinucleotide Suppression Enables Antiviral Defence Targeting Non-Self RNA.” Nature 550 (7674): 124-27. https://doi.org/10.1038/nature24039.

Tan, Bing, Xing-Lou Yang, Xing-Yi Ge, Cheng Peng, Hai-Zhou Liu, Yun-Zhi Zhang, Li-Biao Zhang, and Zheng-Li Shi. 2017. "Novel Bat Adenoviruses with Low G+C Content Shed New Light on the Evolution of Adenoviruses." The Journal of General Virology 98 (4): 739-48. https://doi.org/10.1099/jgv.0.000739.

Taubenberger, Jeffery K., and John C. Kash. 2010. "Influenza Virus Evolution, Host Adaptation, and Pandemic Formation." Cell Host \& Microbe 7 (6): 440-51. https://doi.org/10.1016/j.chom.2010.05.009.

Teeling, E. C., O. Madsen, R. A. Van Den Bussche, W. W. de Jong, M. J. Stanhope, and M. S. Springer. 2002. "Microbat Paraphyly and the Convergent Evolution of a Key Innovation in Old World Rhinolophoid Microbats." Proceedings of the National Academy of Sciences 99 (3): 1431-36. https://doi.org/10.1073/pnas.022477199.

Thain, A., K. Webster, D. Emery, A. R. Clarke, and K. Gaston. 1997. "DNA Binding and Bending by the Human Papillomavirus Type 16 E2 Protein. Recognition of an Extended Binding Site.” The Journal of Biological Chemistry 272 (13): 8236-42. https://doi.org/10.1074/jbc.272.13.8236.

Thierry, F., and M. Yaniv. 1987. "The BPV1-E2 Trans-Acting Protein Can Be Either an Activator or a Repressor of the HPV18 Regulatory Region." The EMBO Journal 6 (11): 3391-97.

Thompson, J, and A Iwasaki. 2008. "Toll-like Receptors Regulation of Viral Infection and Disease 2 ." Advanced Drug Delivery Reviews 60 (7): 786-94. https://doi.org/10.1016/j.addr.2007.11.003.

Thompson, Mikayla R., John J. Kaminski, Evelyn A. Kurt-Jones, and Katherine A. Fitzgerald. 2011. "Pattern Recognition Receptors and the Innate Immune Response to Viral Infection." Viruses 3 (6): 920-40. https://doi.org/10.3390/v3060920. 
1082

1083

1084

1085

1086

1087

1088

1089

1090

1091

1092

1093

1094

1095

1096

1097

1098

1099

1100

1101

1102

1103

1104

1105

1106

1107

1108

1109

1110

1111

1112

1113

1114

1115

1116

1117

1118

Tsagkogeorga, Georgia, Joe Parker, Elia Stupka, James A. Cotton, and Stephen J. Rossiter. 2013. "Phylogenomic Analyses Elucidate the Evolutionary Relationships of Bats." Current Biology 23 (22): 2262-67. https://doi.org/10.1016/j.cub.2013.09.014.

Uhlorn, Brittany L., Eduardo R. Gamez, Shuaizhi Li, and Samuel K. Campos. 2020. "Attenuation of CGAS/STING Activity during Mitosis." Life Science Alliance 3 (9). https://doi.org/10.26508/lsa.201900636.

Uhlorn, Brittany L., Robert Jackson, Shuaizhi Li, Shauna M. Bratton, Koenraad Van Doorslaer, and Samuel K. Campos. 2020. "Vesicular Trafficking Permits Evasion of CGAS/STING Surveillance during Initial Human Papillomavirus Infection.” Edited by Paul Francis Lambert. PLOS Pathogens 16 (11): e1009028. https://doi.org/10.1371/journal.ppat.1009028.

Upadhyay, Mohita, and Perumal Vivekanandan. 2015. "Depletion of CpG Dinucleotides in Papillomaviruses and Polyomaviruses: A Role for Divergent Evolutionary Pressures." Edited by Robert D. Burk. PLOS ONE 10 (11): e0142368. https://doi.org/10.1371/journal.pone.0142368.

Van Doorslaer, Koenraad. 2013. "Evolution of the Papillomaviridae." Virology 445 (1-2): 1120. https://doi.org/10.1016/j.virol.2013.05.012.

Van Doorslaer, Koenraad, Zigui Chen, Hans-Ulrich Bernard, Paul K. S. Chan, Rob DeSalle, Joakim Dillner, Ola Forslund, et al. 2018. "ICTV Virus Taxonomy Profile: Papillomaviridae.” The Journal of General Virology 99 (8): 989-90. https://doi.org/10.1099/jgv.0.001105.

Van Doorslaer, Koenraad, and Joakim Dillner. 2019. "The Launch of an International Animal Papillomavirus Reference Center.” Viruses 11 (1). https://doi.org/10.3390/v11010055.

Van Doorslaer, Koenraad, Zhiwen Li, Sandhya Xirasagar, Piet Maes, David Kaminsky, David Liou, Qiang Sun, Ramandeep Kaur, Yentram Huyen, and Alison A. McBride. 2017. "The Papillomavirus Episteme: A Major Update to the Papillomavirus Sequence Database." Nucleic Acids Research 45 (D1): D499-506. https://doi.org/10.1093/nar/gkw879.

Van Doorslaer, Koenraad, and Alison A. McBride. 2016. "Molecular Archeological Evidence in Support of the Repeated Loss of a Papillomavirus Gene." Scientific Reports 6 (September): 33028. https://doi.org/10.1038/srep33028.

Van Doorslaer, Koenraad, Qina Tan, Sandhya Xirasagar, Sandya Bandaru, Vivek Gopalan, Yasmin Mohamoud, Yentram Huyen, and Alison A. McBride. 2013. "The Papillomavirus Episteme: A Central Resource for Papillomavirus Sequence Data and Analysis." Nucleic Acids Research 41 (Database issue): D571-578. https://doi.org/10.1093/nar/gks984.

Villiers, Ethel-Michele de, Claude Fauquet, Thomas R. Broker, Hans-Ulrich Bernard, and Harald zur Hausen. 2004. "Classification of Papillomaviruses." Virology 324 (1): 17-27. https://doi.org/10.1016/j.virol.2004.03.033.

Vincent, Isabelle E., Claudia Zannetti, Julie Lucifora, Helene Norder, Ulrike Protzer, Pierre Hainaut, Fabien Zoulim, et al. 2011. "Hepatitis B Virus Impairs TLR9 Expression and Function in Plasmacytoid Dendritic Cells." PloS One 6 (10): e26315. https://doi.org/10.1371/journal.pone.0026315.

Vinokurova, Svetlana, and Magnus von Knebel Doeberitz. 2011. "Differential Methylation of the HPV 16 Upstream Regulatory Region during Epithelial Differentiation and Neoplastic Transformation.” Edited by Torbjorn Ramqvist. PLoS ONE 6 (9): e24451. https://doi.org/10.1371/journal.pone.0024451. 
Wacharapluesadee, Supaporn, Chee Wah Tan, Patarapol Maneeorn, Prateep Duengkae, Feng Zhu, Yutthana Joyjinda, Thongchai Kaewpom, et al. 2021. "Evidence for SARS-CoV-2 Related Coronaviruses Circulating in Bats and Pangolins in Southeast Asia." Nature Communications 12 (1): 972. https://doi.org/10.1038/s41467-021-21240-1. 
1163 Yu, Guangchuang, Tommy Tsan-Yuk Lam, Huachen Zhu, and Yi Guan. 2018. "Two Methods 1164 for Mapping and Visualizing Associated Data on Phylogeny Using Ggtree." Molecular Biology and Evolution 35 (12): 3041-43. https://doi.org/10.1093/molbev/msy194. Xiaodong Fang, James W. Wynne, et al. 2013. "Comparative Analysis of Bat Genomes Provides Insight into the Evolution of Flight and Immunity." Science (New York, N.Y.) 339 (6118): 456-60. https://doi.org/10.1126/science.1230835. Akira, Lena Alexopoulou, and Marc Dalod. 2008. "Cutting Edge: Overlapping Functions of TLR7 and TLR9 for Innate Defense against a Herpesvirus Infection." The Journal of Immunology 180 (9): 5799-5803. https://doi.org/10.4049/jimmunol.180.9.5799. 


\section{Figure Legends}

\section{Figure 1 Evolutionary relationship of novel bat papillomaviruses}

1178 (A) Maximum-likelihood phylogenetic tree inferred using concatenated E1, E2, and L1 protein 1179 sequences. Papillomaviruses associated with Chiroptera are highlighted Yangochiroptera 1180 (orange) and Yinpterochiroptera (red). Papillomavirus genera are collapsed (number of 1181 types within each genus are indicated in parentheses). Bootstrap generated branch 1182 support values are given using symbols and color gradient. Host species are indicated 1183 using Sonoran Desert dwelling animals. The red arrow indicates the subtree used for 1184 further analyses throughout the manuscript.

1185 (B) Pairwise identity plot with percentage pairwise identities provided in colored boxes for the L1 nucleotide sequences.

(A) Optimized tanglegram between subtree based on concatenated E1-E2-L1 maximumlikelihood phylogenetic tree (see Figure 1) and associated host species. Host species tree was downloaded from www.timetree.org. Papillomaviruses are linked to their host phylogenies. Papillomaviruses associated with Chiroptera are highlighted Yangochiroptera (orange) and Yinpterochiroptera (blue).

(B) Procrustean Approach to Cophylogeny analysis based on the interaction network and phylogenies shown in (A) supports that papillomaviruses coevolved with their hosts. The observed best-fit Procrustean super-imposition (red dotted line line) lies outside of the 95\% confidence interval (shaded area of the curves) of the distribution of network randomizations in the null model. 
1202 Figure 3 CpG dinucleotide sequences are significantly depleted in papillomavirus 1203 genomes

1204 The observed vs. expected (O/E) ratios of each dinucleotide in the papillomavirus genomes

1205 sequences shown in Figure 2 were calculated using a custom wrapper around the CompSeq

1206 program from the EMBOSS software suite. The red line indicates that the sequence is seen as

1207 often as would be expected by chance.

Figure $4 \mathrm{CpG}$ content is significantly lower in papillomaviruses associated with 1210 Yangochiroptera compared to related viruses

1211 (A) A maximum likelihood phylogenetic tree is shown comparing the O/E ratios of CpG 1212 dinucleotides. Viruses infecting Yangochiroptera (red), Yinpterochiroptera (green), and 1213 related hosts (grey) are indicated.

(B) Mean (+/- standard deviation) CpG observed vs. expected (O/E) ratios for each group of viruses are compared using a one-way ANOVA with Tukey's posthoc test.

(A) Codon usage tables for each virus in Figure 2 were compared using the 'codcmp' program from the EMBOSS software suite. Root-mean-square deviation (RMSD) values for each pairwise comparison are plotted as Box-and-whisker plots with the outliers (colored circles) identified using Tukey’s method. Individual values are shown as a single black dot.

(B) Amino-acid composition was calculated as described in materials and methods. Mean values +/- standard deviation is plotted.

(C) RSCU values for the indicated amino acid/codons were calculated and plotted as Boxand-whisker plots with the outliers (colored circles) identified using Tukey's method. RSCU 
values for each amino acid were compared using a two-way ANOVA with Tukey's posthoc test. Significance is indicated as shown in the legend.

Figure 6 Yangochiroptera TLR9 is evolving under diversifying selection

(A) Structure of horse TLR9 in complex with agonistic DNA (PDB: 3WPC) (Ohto et al. 2015). Amino acids of interest are highlighted.

(B) Maximum likelihood phylogenetic tree of mammalian TLR9 sequences clusters Yangochiroptera and Yinpterochiroptera separate from the mammalian TLR9. Red branches display evidence of episodic diversifying selection as identified by aBRSEL (Smith et al. 2015). Alignments show sequences of interest. The sequence logo is based on the alignment of 29 non-chiropteran TLR9 sequences. Numbering is based on the mouse TLR9. Residues indicated with $\$$ were identified as being selected using FEL. Residues highlighted with * were previously identified as evolving under diversifying selection (Escalera-Zamudio et al. 2015), while residues with \# were shown to be functionally important through site directed mutagenesis (Ohto et al. 2015.

\section{5 their genomes}

1246 (A) The observed vs. expected (O/E) ratios of each N-CG-N tetramer in the Yangochiroptera 1247 papillomavirus genomes sequences were calculated using a custom wrapper around the 1248 CompSeq program from the EMBOSS software suite. Mean values +/- standard deviation 1249 are plotted.

1250 (B) The observed vs. expected (O/E) ratios of each N-CG-N tetramer in the different groups 1251 was calculated as in A. The proportion of these ratios are shown to provide a normalized 1252 view of tetramer depletion across papillomavirus genomes shown in Figure 2. 
bioRxiv preprint doi: https://doi.org/10.1101/2021.04.17.440006; this version posted April 18, 2021. The copyright holder for this preprint (which

was not certified by peer review) is the author/funder, who has granted bioRxiv a license to display the preprint in perpetuity. It is made available under aCC-BY 4.0 International license.

1253 (C) The Yang vs. Yin N-CG-N proportion (as in B) are plotted as brown dots and compared to

12541000 randomly shuffled sequences (green violin) plots. Only ACGT is statistically 1255 underrepresented in the Yangochiroptera.

1256

1257 
bioRxiv preprint doi: https://doi.org/10.1101/2021.04.17.440006; this version posted April 18, 2021. The copyright holder for this preprint (which was not certified by peer review) is the author/funder, who has granted bioRxiv a license to display the preprint in perpetuity. It is made

- Alphapapillomainable under aCC-BY 4.0 International license.
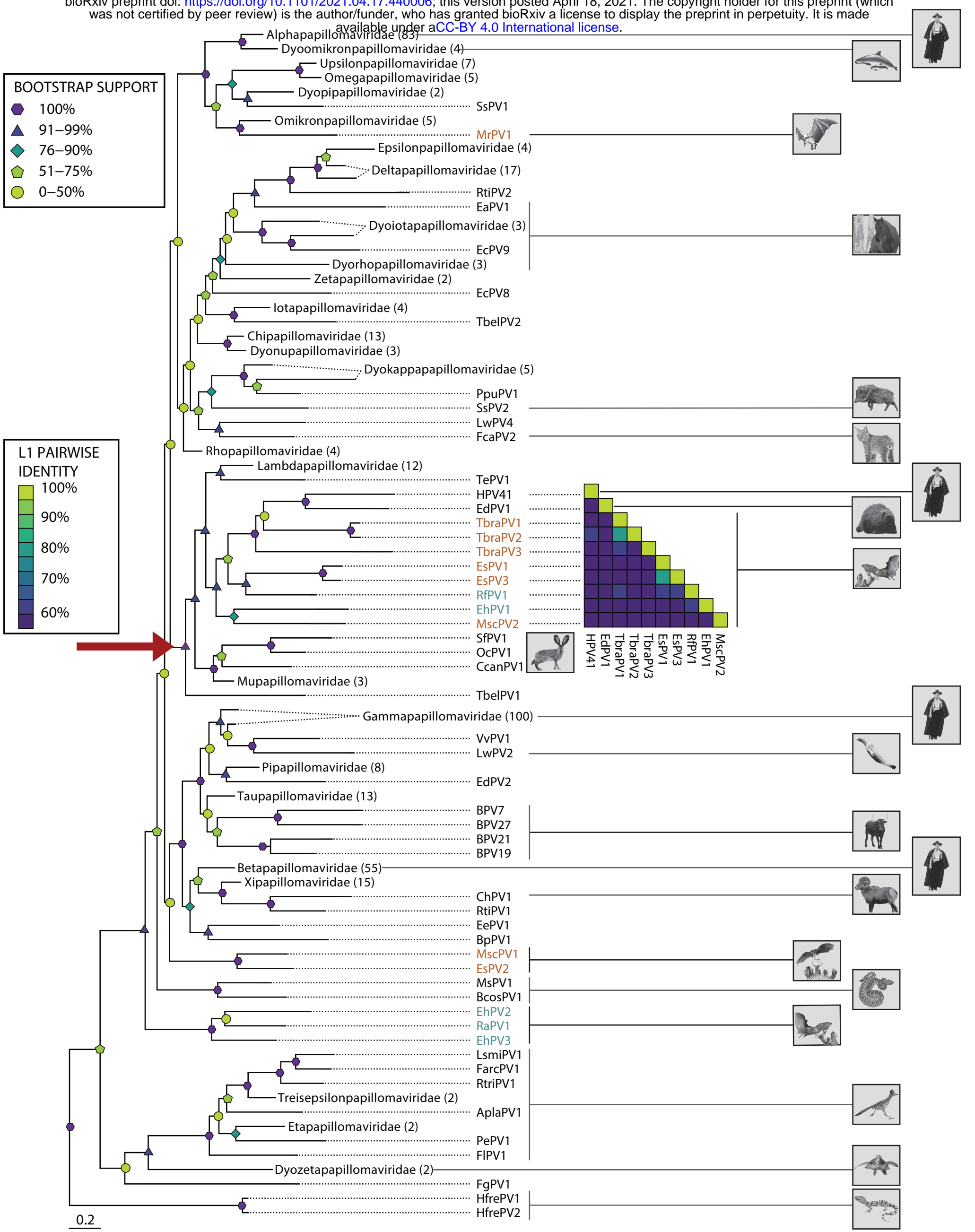
bioRxiv preprint doi: https://doi.org/10.1101/2021.04.17.440006; this version posted April 18, 2021. The copyright holder for this preprint (which

was not certified by peer review) is the author/funder, who has granted bioRxiv a license to display the preprint in perpetuity. It is made

A.
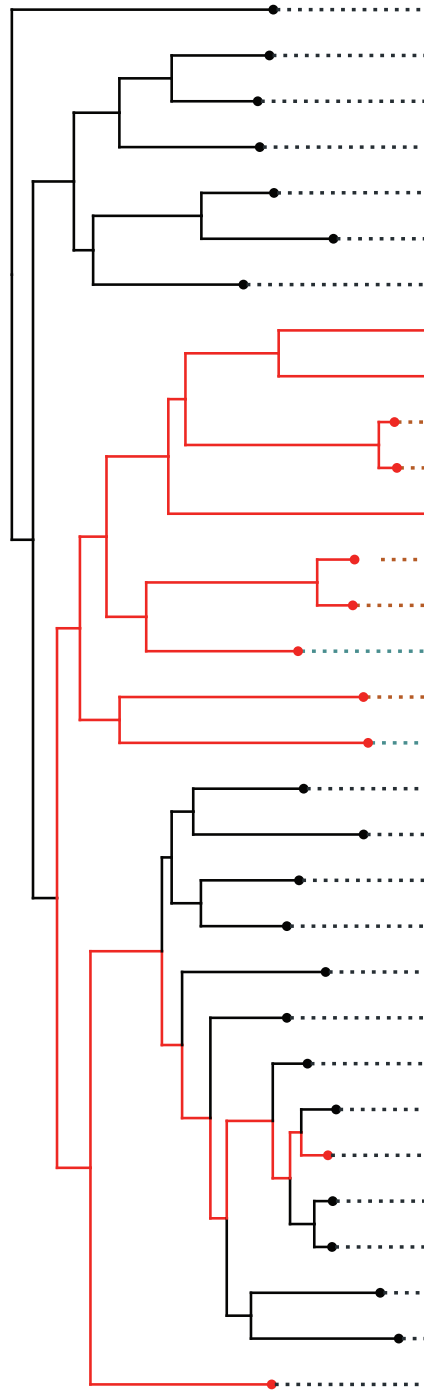

TbelPV1

HPV1

HPV63

HPV204

OcPV1

SfPV1

CcanPV1

HPV41

EdPV1

TbraPV1

TbraPV2

TbraPV3

EsPV1

EsPV3

RfPV1

$\mathrm{MscPV} 2$

EhPV1

AmPV4

LwPV1

EIPV1

PIPV1

CPV1

CPV6

FcaPV1

PcPV1

LrPV1

UuPV1

PIpPV1

LwiePV1

CcrPV1

TePV1
B.

,
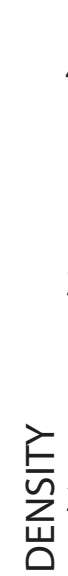
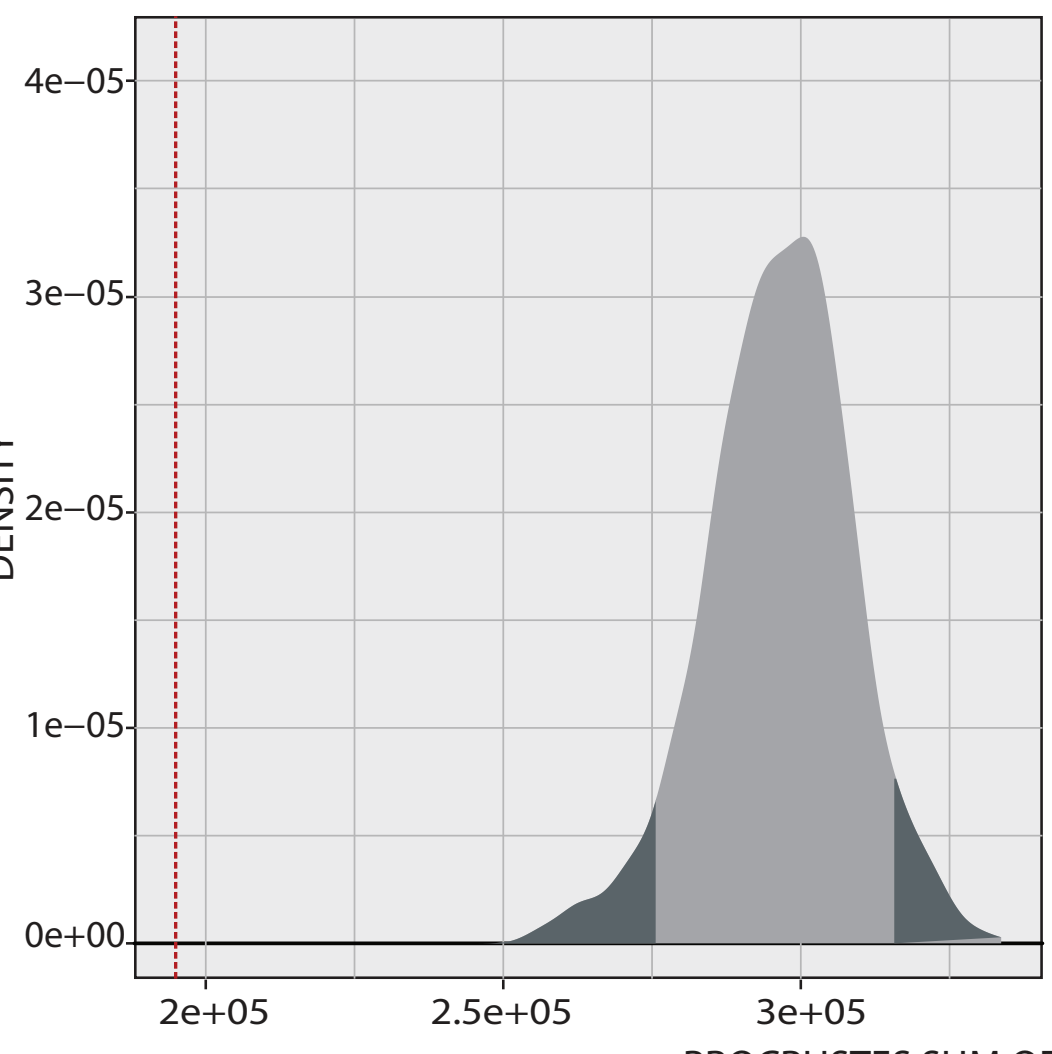

Tupaia belangeri

Homo sapiens

Oryctolagus cuniculus

Sylvilagus floridanus

Castor canadensis

Erethizon dorsatum

Tadarida brasiliensis

Eptesicus serotinus

Miniopterus schreibersii

Rhinolophus ferrumequinum

Eidolon helvum

Ailuropoda melanoleuca

Leptonychotes weddellii

Enhydra lutris

Procyon lotor

Canis familiaris

Puma concolor

Lynx rufus

Felis domesticus

Leopardus wiedii

Uncia uncia

Panthera leo persica

Crocuta crocuta

$\underline{\text { Talpa europaea }}$

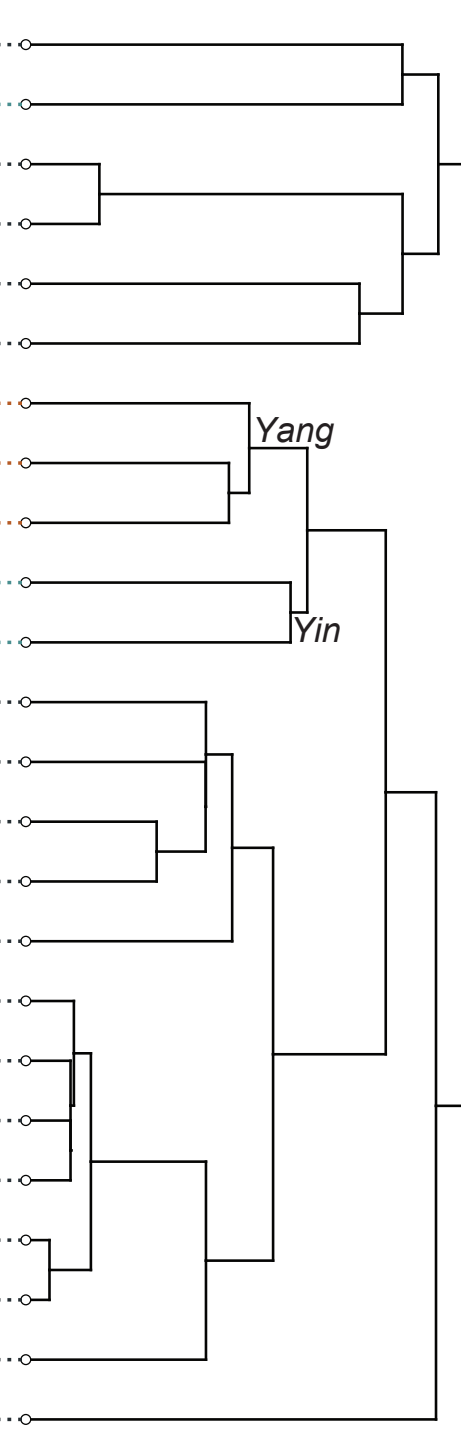

C.

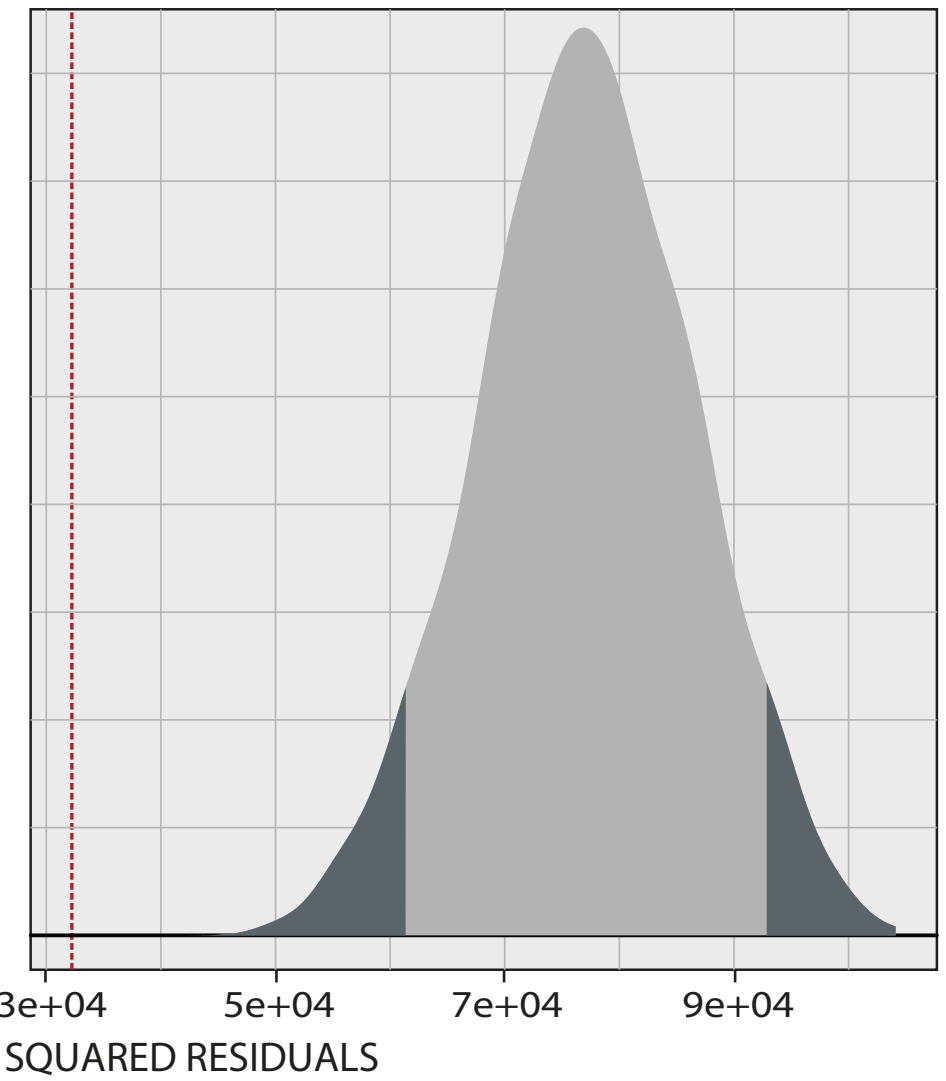


bioRxiv preprint doi: https://doi.org/10.1101/2021.04.17.440006; this version posted April 18, 2021. The copyright holder for this preprint (which was not certified by peer review) is the author/funder, who has granted bioRxiv a license to display the preprint in perpetuity. It is made available under aCC-BY 4.0 International license.

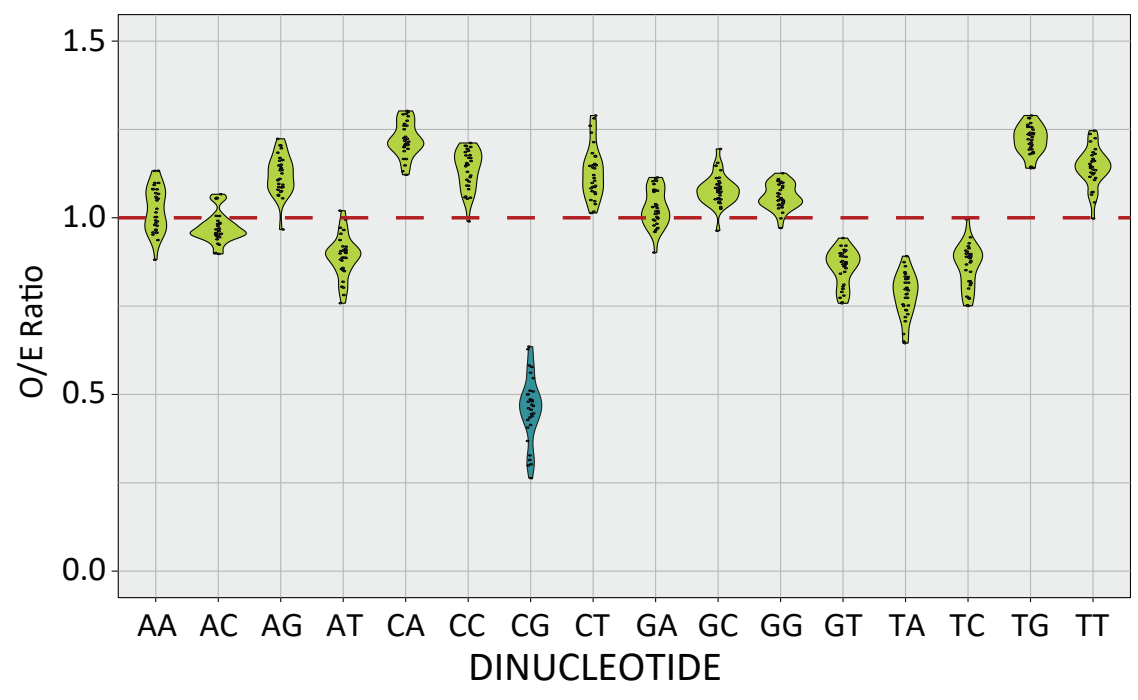


bioRxiv preprint doi: https://doi.org/10.1101/2021.04.17.440006; this version posted April 18, 2021. The copyright holder for this preprint (which was not certified by peer review) is the author/funder, who has granted bioRxiv a license to display the preprint in perpetuity. It is made

A.

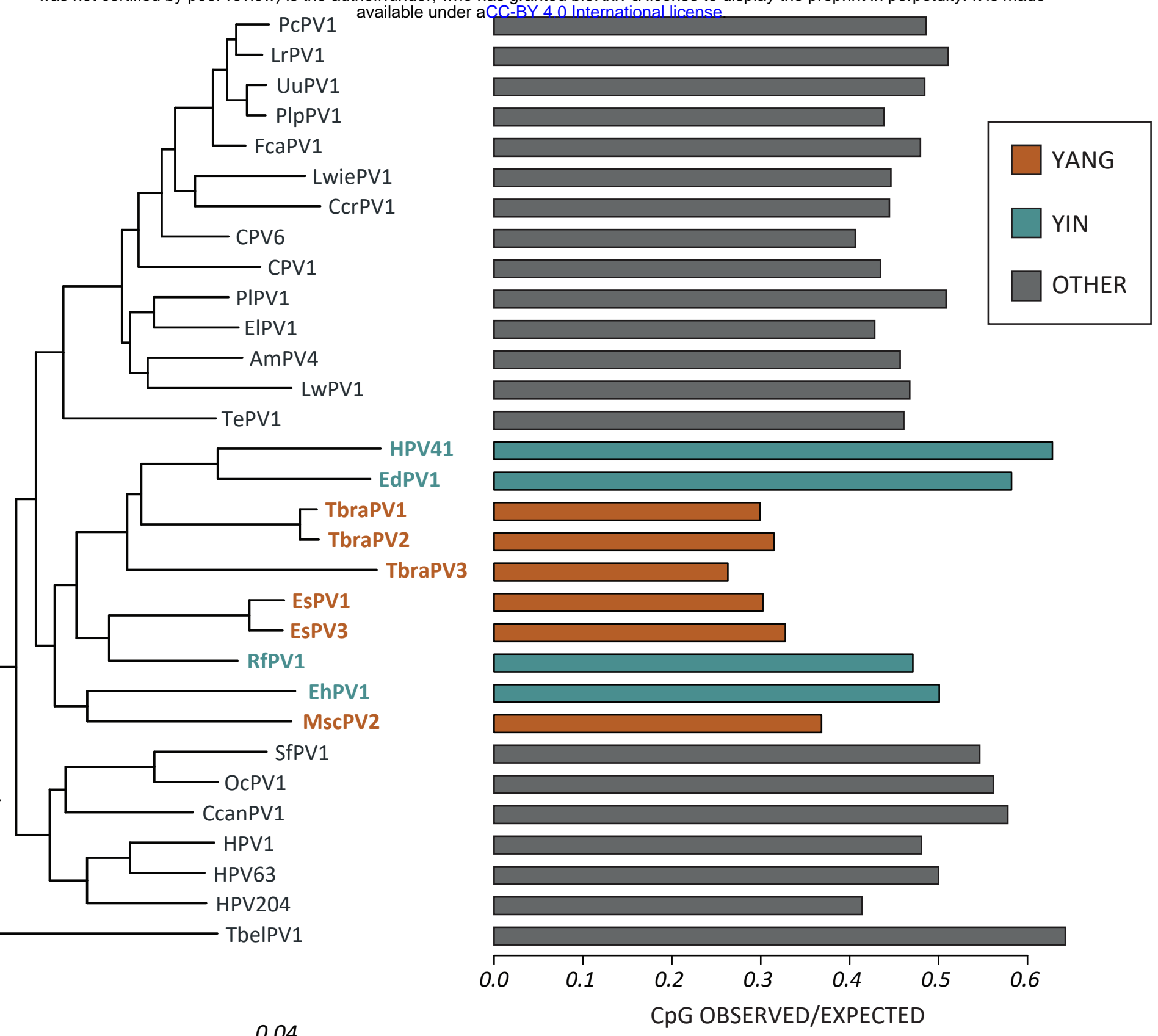

B.

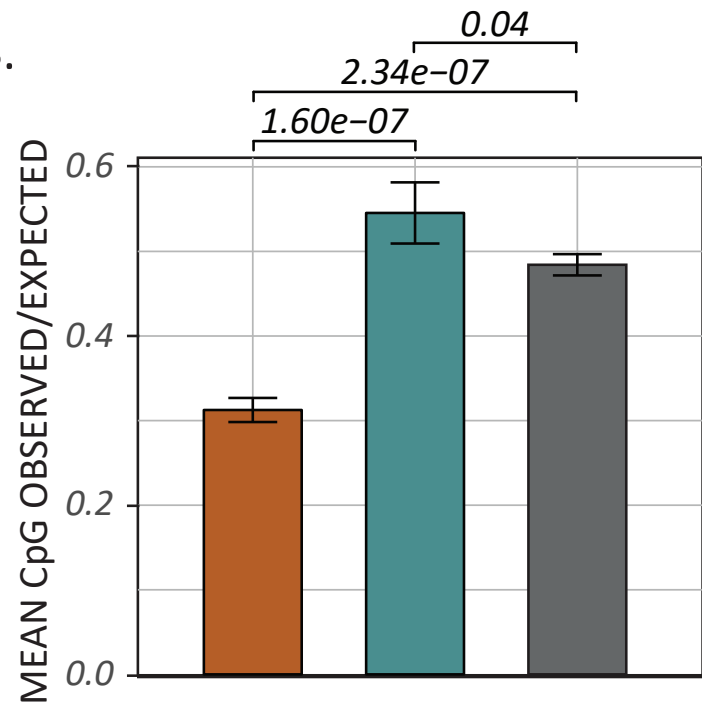

C.

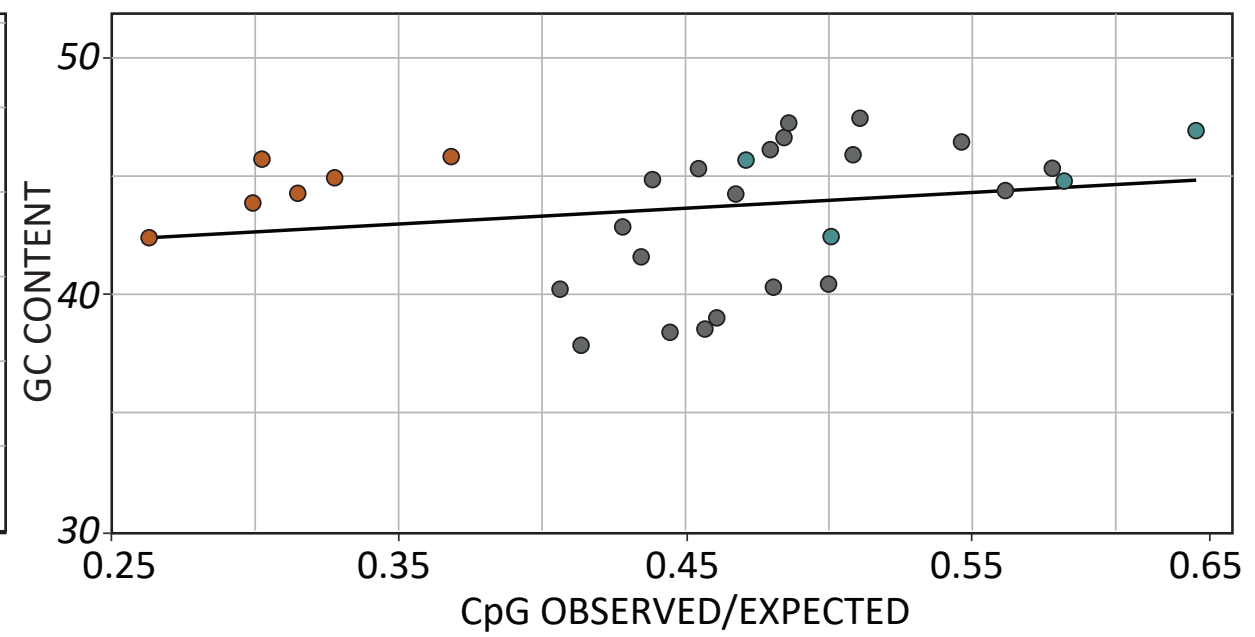



bioRxiv preprint doi: https://doi.org/10.1101/2021.04.17.440006; this version posted April 18, 2021. The copyright holder for this preprint (which
was not certified by peer review) is the author/funder, who has granted bioRxiv a license to display the preprint in perpetuity. It is made
available under aCC-BY 4.0 Int $R$ (national license.

A.

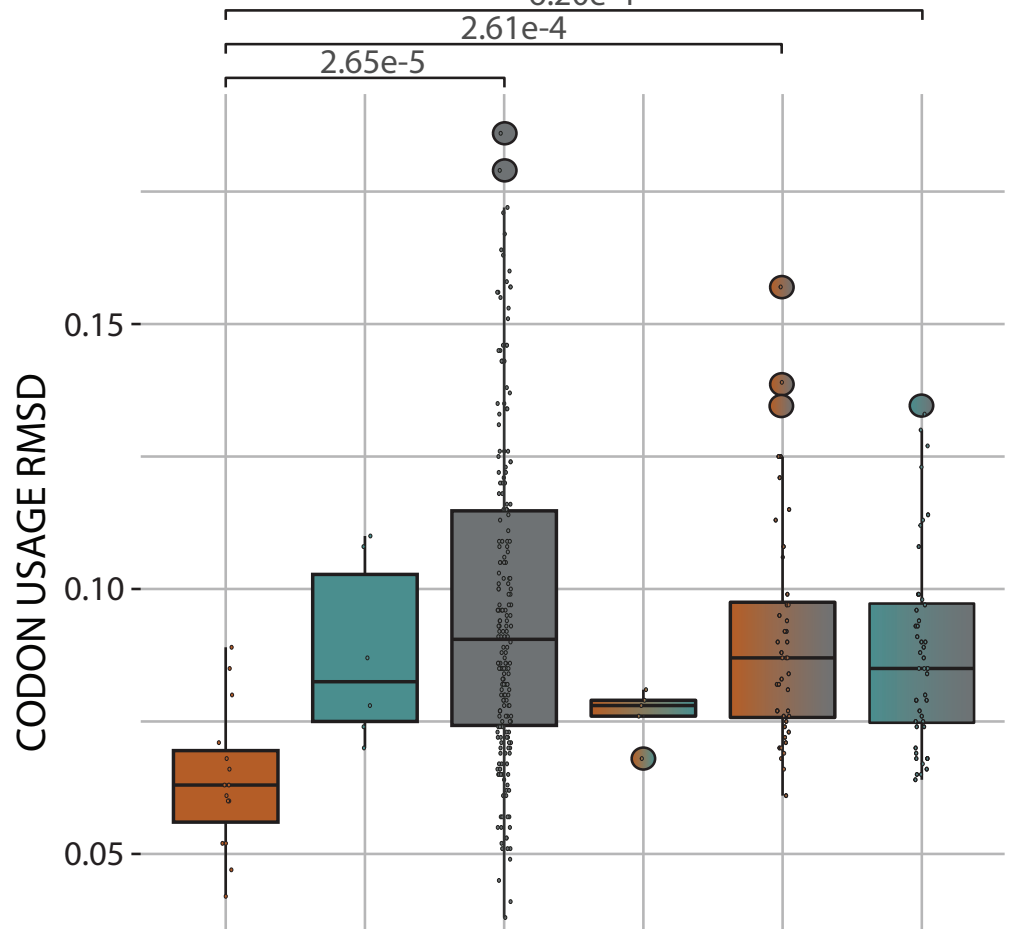

YANG - YIN+ - OTHER - YANG - OTHER - OTHER YANG YIN+ OTHER YIN+ YANG YIN+

C.

\begin{tabular}{|c|c|c|}
\hline$*$ & $* *$ & $* * *$ \\
\hline$<0.05$ & $<0.01$ & $<0.001$ \\
\hline
\end{tabular}
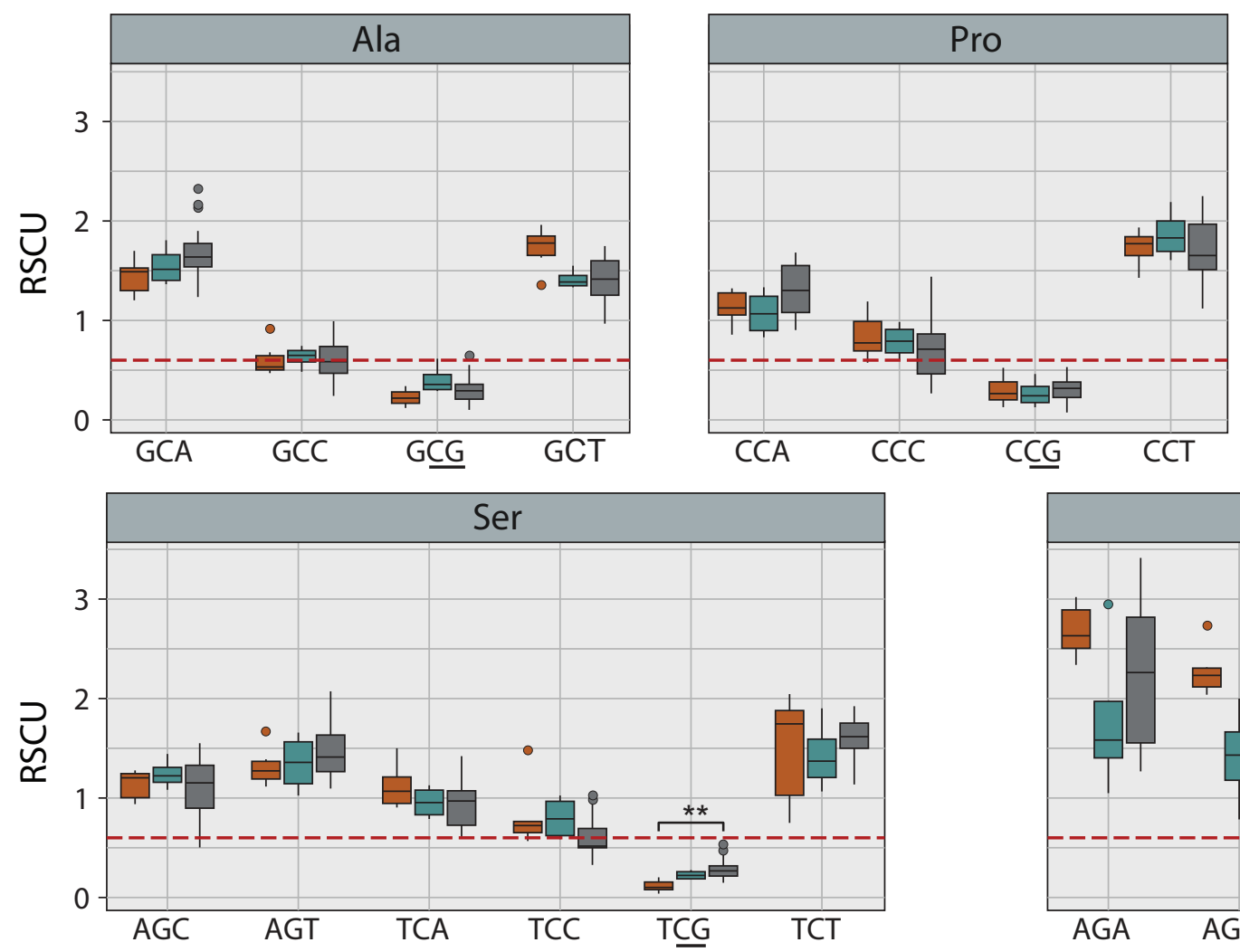

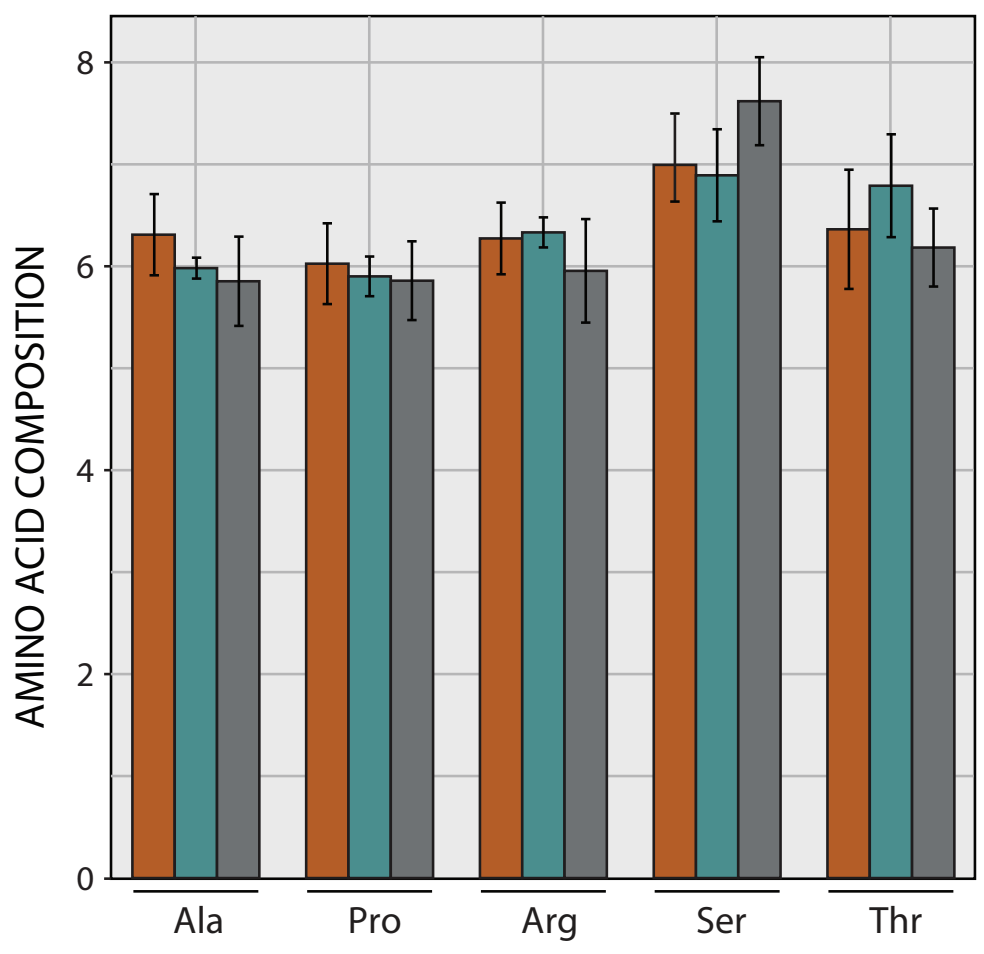

YANG $\square$ YIN $\square$ OTHER
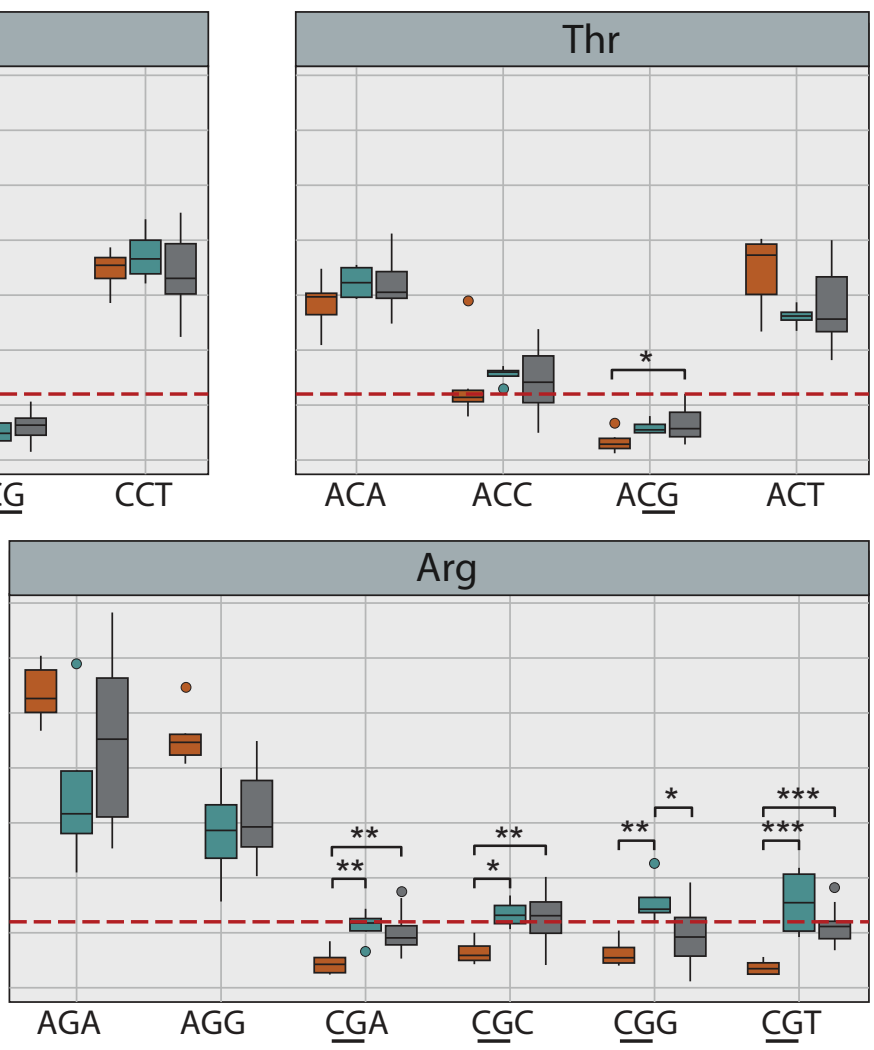
bioRxiv preprint doi: https://doi.org/10.1101/2021.04.17.440006; this version posted April 18, 2021. The copyright holder for this preprint (which

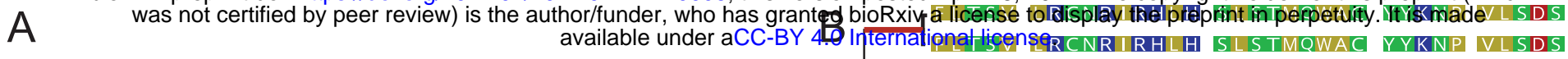
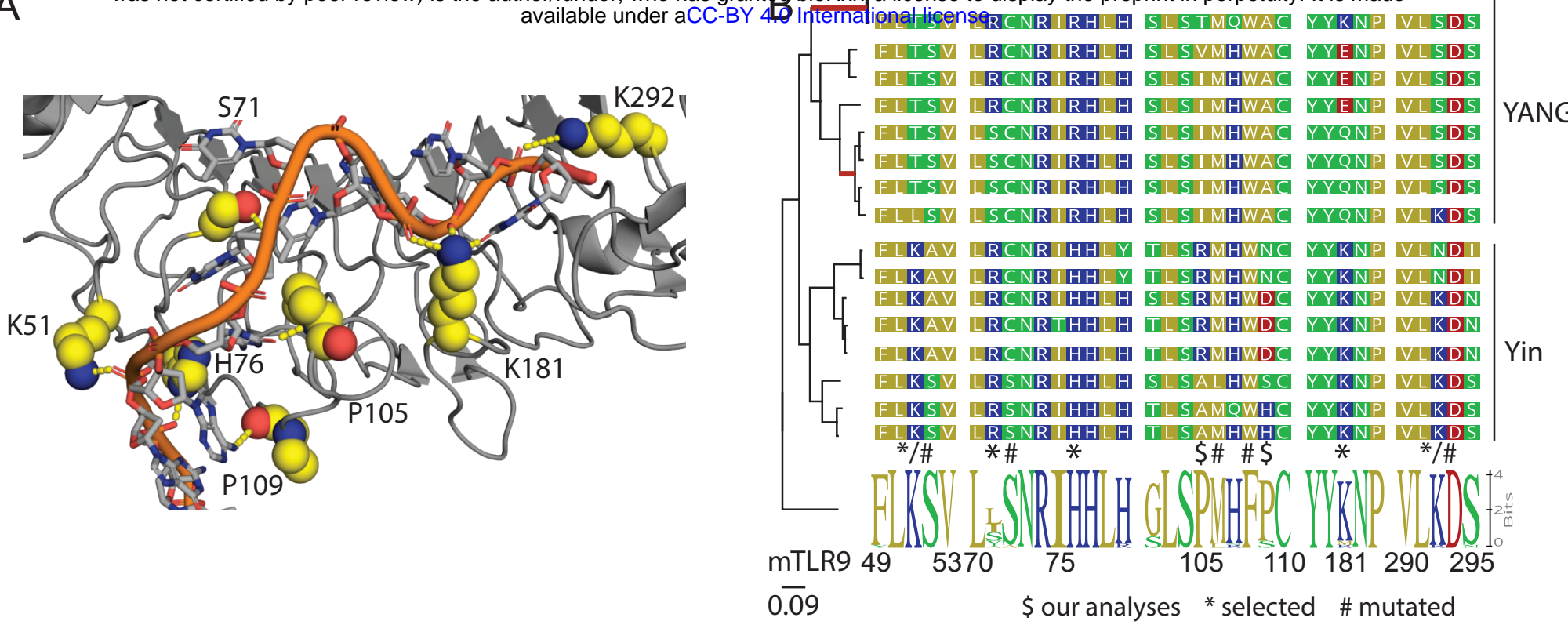
A. was not certified by peer review) is the author/funder, who has granted bioRxiv a license to display the preprint in perpetuity. It is made available under aCC-BY 4.0 International license.

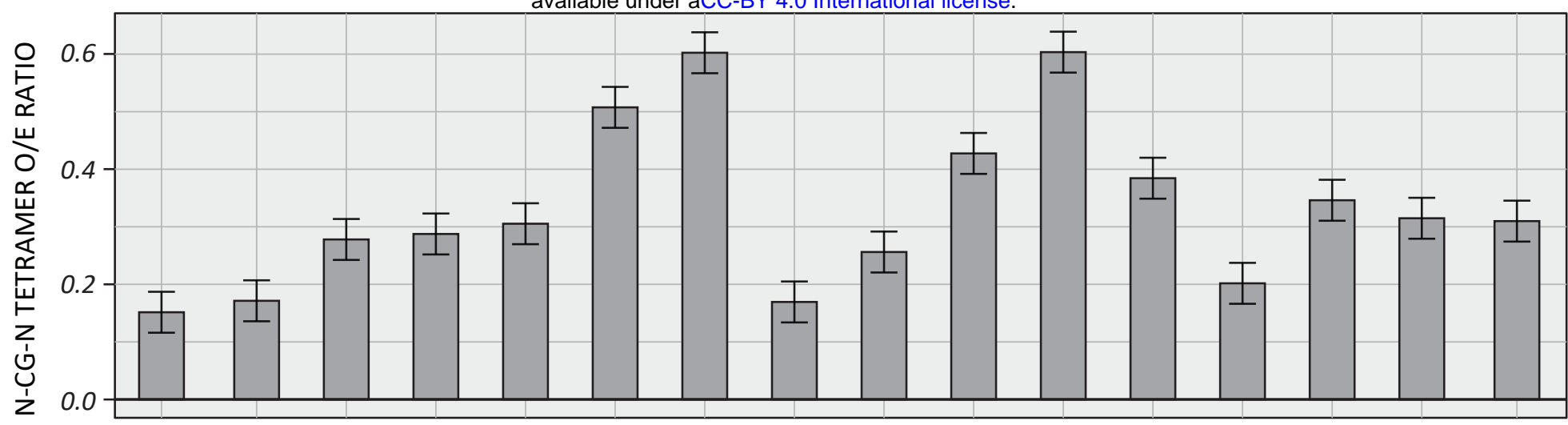

B.

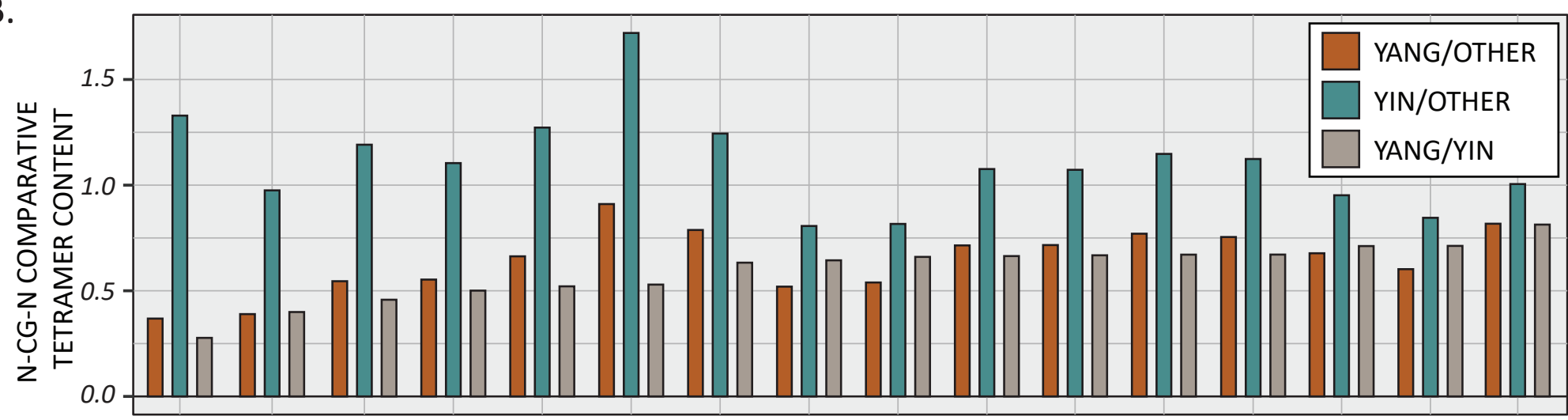

C.

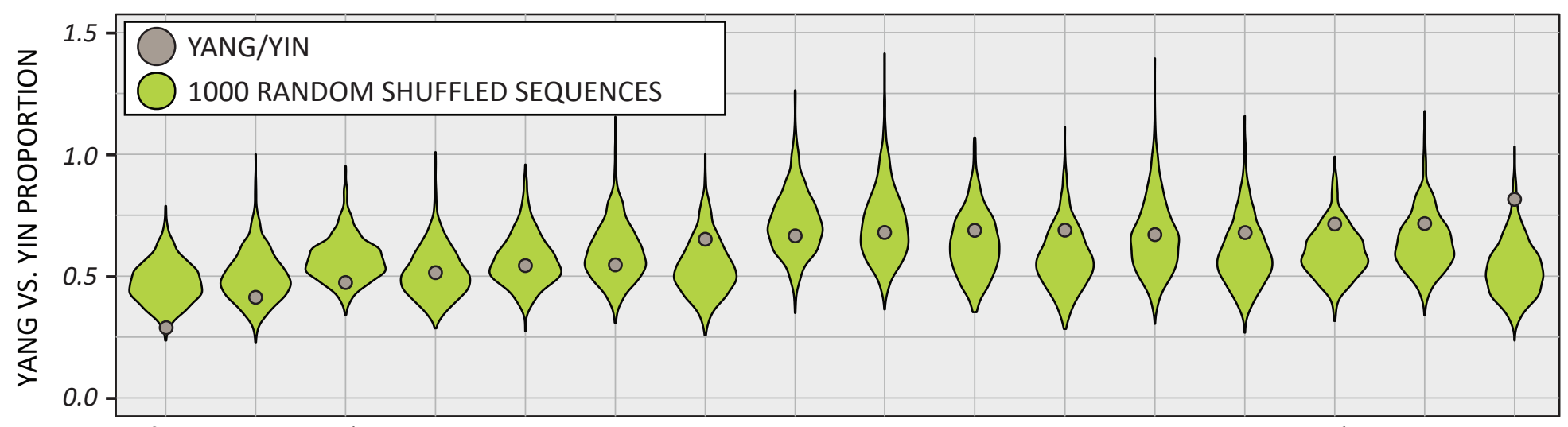

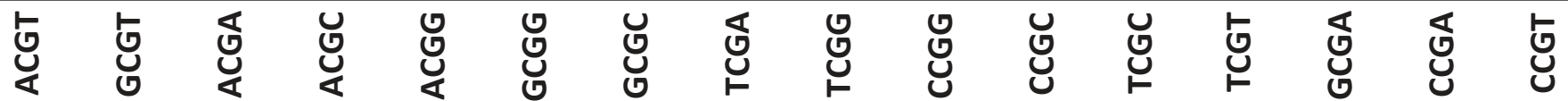

